\title{
NONLINEAR VOLTERRA INTEGRODIFFERENTIAL EVOLUTION INCLUSIONS AND OPTIMAL CONTROL*
}

\author{
By Nikolaos S. Papageorgiou**
}

\begin{abstract}
In this paper we examine integrodifferential evolution inclusions of the Volterra type driven by time dependent, monotone, hemicontinuous operators. We prove two existence theorems; one for convex valued perturbations and the other for nonconvex valued ones. We also establish a topological property of the solution set of the "convex" problem. Then we prove a result on the continuous dependence of the solutions on the data of the problem (sensitivity analysis). We also consider a random version of the inclusion and prove that it admits a random solution. Then we pass to optimal control problems. First we establish the existence of optimal admissible pairs and then using the notions of epigraphical and G-convergences, we obtain a variational stability result. Finally we work in detail two parabolic distributed parameter optimal control problems, illustrating the applicability of our work.
\end{abstract}

\section{List of symbols}

$\Omega:$ Upper case Greek letter omega

$W_{r}: W$ subscript $r$

$\Sigma$ : Upper case Greek letter sigma

$\mathscr{Q}:$ Script $P$

$S^{q}: S$ subscript $q$

$S_{F}^{p}: S$ superscript $p$, subscript $F$

$\omega$ : Lower case Greek letter omega

$W_{p q}: W$ subscript $p q$

$L^{q}: L$ superscript $q$

$L^{p}: L$ superscript $p$

$\tau$ : Lower case Greek letter tau

$\varepsilon$ : Lower case Greek letter epsilon

$\eta$ : Lower case Greek letter eta

$\mathcal{L}:$ Script $L$

$d_{H}: d$ subscript $H$

$\phi:$ Lower case Greek letter phi

$\phi$ : Lower case Greek letter psi

$\delta$ : Lower case Greek letter delta

$B_{M}: B$ subscript $M$

$\alpha$ : Lower case Greek letter alpha

$\beta$ : Lower case Greek letter beta

$\gamma$ : Lower case Greek letter gamma

$\Gamma$ : Upper case Greek letter gamma

$a_{\imath \jmath}^{n}: a$ superscript $n$, subscript ij

\section{Introduction}

In this paper we study Volterra integrodifferential evolution inclusions of nonconvolution type with time dependent unbounded operators and with both

* Revised version.

** Research supported by NSF Grant DMS-8802688.

Received April 10, 1990; revised December 5, 1990. 
convex and nonconvex multivalued perturbations. Our results are then applied to distributed parameter optimal control problems. Our work extends those of Chuong [9], Glashoff-Sprekels [15], Kiffe [16], Papageorgiou [27], [28], Ragimkhanov [32] (on integral inclusions) and Angell [1], Cesari [8], Warga [39] (on optimal control problems), since we allow for the presence of time varying, unbounded nonlinear operators and also our system is an integrodifferential equation. Furthermore for both our inclusion and optimal control problems we conduct a detailed sensitivity (i. e. dependence on the data) analysis.

The structure of the paper is the following. In the next section we establish our notation and terminology and we recall some basic definitions and facts from nonlinear analysis and the theory of measurable multifunctions. In section 3 we pass to the study of the integrodifferential evolution inclusion and we have two existence results; one for convex valued perturbations and the other for nonconvex valued ones. Also in the "convex case", we prove the solution set is compact in an appropriate topology. In section 4 we examine the dependence of the solutions on the data that determine them, namely the unbounded operator, the multivalued perturbation and the initial condition (sensitivity analysis). In section 5 we consider a random version of the integrodifferential evolution inclusion and using techniques from the theory of measurable multifunctions, we establish the existence of random solutions. Our result (theorem 5.1) extends earlier ones obtained by Chuong [9], Papageorgiou [29] and Tsokos-Padgett [37]. In section 6 we turn our attention to optimal control problems for infinite dimensional systems governed by integrodifferential evolution equations of the Volterra type (i. e. systems with memory). We establish the existence of optimal controls and we also conduct a sensitivity analysis using the notions of $\tau$-convergence (epiconvergence) of functions and of $G$-convergence of operators. Finally in section 7 we work in detail two examples of distributed parameter control systems governed by parabolic integrodifferential equations.

\section{Preliminaries}

Let $(\Omega, \Sigma)$ be a measurable space and $X$ a separable Banach space. Throughout this paper we will be using the following notations:

and

$$
P_{f(c)}(X)=\{A \subseteq X: \text { nonempty, closed, (convex) }\}
$$

$$
P_{(w) k(c)}(X)=\{A \subseteq X \text { : nonempty, (weakly-) compact, (convex) }\} \text {. }
$$

A multifunction $F: \Omega \rightarrow P_{f}(X)$ is said to be measurable (see Wagner [38]), if for every $x \in X, \omega \rightarrow d(x, F(\omega))=\inf \{\|x-z\|: z \in F(\omega)\}$ is measurable. A multifunction $F: \Omega \rightarrow 2^{X} \backslash\{\varnothing\}$ is said to be "graph measurable", if $G r F=\{(\omega, x) \in$ $\Omega x X: x \in F(\omega)\} \in \Sigma x B(X)$, with $B(X)$ being the Borel $\sigma$-field of $X$. For $P_{f}(X)$ valued multifunctions measurability implies graph measurability. The converse is true if there is a $\sigma$-finite measure $\mu(\cdot)$ on $\Sigma$ and $\Sigma$ is $\mu$-complete. By $S_{F}^{p}(1 \leqq$ $p \leqq \infty)$ we will denote the set of measurable selectors of $F(\cdot)$ that belong in 
the Lebesgue-Bochner space $L^{p}(X)$; i. e. $S_{F}^{p}=\left\{f \in L^{p}(X): f(\boldsymbol{\omega}) \in F(\omega) \mu\right.$-a.e. $\}$. This set may be empty. For a graph measurable multifunction $F: \Omega \rightarrow 2^{X} \backslash\{\varnothing\}$, $S_{F}^{p}$ is nonempty if and only if $\omega \rightarrow \inf \{\|z\|: z \in F(\omega)\} \in L_{+}^{p}$. In particular this is the case if $\omega \rightarrow|F(\omega)|=\sup \{\|z\|: z \in F(\omega)\} \in L_{+}^{p}$. Such a multifunction is said to be $L^{p}$-integrably bounded. Using $S_{F}^{1}$ we can define a set valued integral for $F(\cdot)$ by setting $\int_{\Omega} F(\omega) d \mu(\omega)=\left\{\int_{\Omega} f(\omega) d \mu(\omega): f \in S_{F}^{1}\right\}$.

Let $Y, Z$ be Hausdorff topological spaces and $G: Y \rightarrow 2^{Z} \backslash\{\varnothing\}$ a multifunction. We say that $G(\cdot)$ is upper semicontinuous (u. s. c.) (resp. lower semicontinuous (l. s. c.)), if for all $V \subseteq Z$ open, the set $G^{+}(V)=\{y \in Y: G(y) \subseteq V\}$ (resp. $G^{-}(V)=$ $\{y \in Y: G(y) \cap V \neq \varnothing\})$ is open in $Y$. If $Z$ is a metric space, then on $P_{f}(Z)$ we can define a (generalized) metric, known in the literature as the Hausdorff metric, by setting

$$
h(A, B)=\max \left\{\sup _{a \in A} d(a, B), \sup _{b \in B} d(b, A)\right\}
$$

If $Z$ is complete, then so is the metric space $\left(P_{f}(Z), h\right)$.

Finally let $T=[0, r], H$ a separable Hilbert space and $X$ a subspace of $H$ carrying the structure of a separable, reflexive Banach space which is continuously and densely embedded into $H\left(\right.$ i. e. $\left.X \varsigma_{\rightarrow} H\right)$. Having $H$ as our pivot space, we get $X \hookrightarrow H \subset{ }^{*}$. Such a triple of spaces $\left(X, H, X^{*}\right)$ is known in the literature as "Gelfand triple" or "spaces in normal position". By $\|\cdot\|$ (resp. $|\cdot|$, $\left.\|\cdot\|_{*}\right)$ we will denote the norm of $X$ (resp. of $\left.H, X^{*}\right)$. Also by $\langle\cdot, \cdot\rangle$ we will denote the duality crackets for the pair $\left(X, X^{*}\right)$ and by $(\cdot, \cdot)$ the inner product of $H$. The two are compatible in the sense that $\left\langle\cdot,\left.\cdot \cdot\right|_{x_{\times H}}=(\cdot, \cdot)\right.$. By $W_{p q}(T)$ we will denote the space of elements $x \in L^{p}(X)$ such that the distributional derivative $\dot{x} \in L^{q}\left(X^{*}\right)$. $p^{-1}+q^{-1}=1$ Furnished with the norm

$$
\|x\|_{W_{p q}(T)}=\left\{\|x\|_{L^{2}(X)}^{2}+\|\dot{x}\|_{\left.L^{2} q_{\left(X^{*}\right)}\right\}^{1 / 2},}\right.
$$

$W_{p q}(T)$ becomes a separable Banach space (reflexive if $p>1$ ) and it is well known that $W_{p q}(T)$ can be embedded in the Banach space $C(T, H)$; i. e. $W_{p q}(T) \hookrightarrow$ $C(T, H)$. So every element in $W_{p q}(T)$, after possible modification on a Lebesguenull subset of $T$ is equal to an $H$-valued continuous function defined on $T$. When $X \hookrightarrow H$ compactly, from theorem 5.1, p. 58 of Lions [22], we have that $W_{p q}(T) \hookrightarrow L^{p}(H)$ compactly. When $p=q=2$, we will simply write $W(T)$.

\section{Integrodifferential inclusions-existence theorems}

Let $T=[0, r]$ and $\left(X, H, X^{*}\right)$ a Gelfand triple of spaces, with all embeddings assumed to be compact. This setting will remain in effect for the rest of this paper. We will be studying the following Volterra integrodifferential evolution inclusion :

$$
\left\{\begin{array}{l}
\dot{x}(t)+A(t, x(t)) \in \int_{0}^{t} k(t-s) F(s, x(s)) d s \quad \text { a. e. on } T \\
x(0)=x_{0}
\end{array}\right\}\left(^{*}\right)
$$


By a solution of $(*)$, we understand a function $x(\cdot) \in W_{p q}(T)$ s. t. there exists $f(\cdot) \in S_{F(\cdot, x(\cdot))}^{q}$ for which we have $\dot{x}(t)+A(t, x(t))=\int_{0}^{t} k(t-s) f(s) d s$ a. e. in $X^{*}$ and $x(0)=x_{\theta}$.

We will need the following hypotheses on the data:

$H(A): A: T x X \rightarrow X^{*}$ is an operator s. t.

(1) $t \rightarrow A(t, x)$ is measurable,

(2) $x \rightarrow A(t, x)$ is hemicontinuous, monotone,

(3) $\|A(t, x)\|_{*} \leqq c\left(1+\|x\|^{p-1}\right)$ a. e. with $c>0$ and $p \geqq 2$,

(4) $\langle A(t, x), x\rangle \geqq c_{1}\|x\|^{p}$ a. e. with $\left.c_{1}\right\rangle 0$.

$H(F): F: T x H \rightarrow P_{f c}(H)$ is a multifunction s. t.

(1) $F(\cdot, \cdot)$ is graph measurable,

(2) $F(t, \cdot)$ has a graph which is sequentially closed in $H \times H_{w}$ where $H_{w}$ denotes the Hilbert space $H$ with the weak topology.

(3) $|F(t, x)| \leqq a(t)+b|x|^{2 / q}$ a. e. with $a(\cdot) \in L_{+}^{q}, b>0$, where $\frac{1}{p}+\frac{1}{q}=1$.

$H(k): k \in L^{\infty}(T, \mathcal{L}(H))$.

THEOREM 3.1. If hypotheses $H(A), H(F), H(k)$ hold, then (*) admits a solution.

Proof. First we will obtain an a priori bound for the solutions of $\left(^{*}\right)$. So let $x(\cdot) \in W_{p q}(T)$. By definition we have:

$$
\begin{aligned}
& \dot{x}(t)+A(t, x(t))=\int_{0}^{t} k(t-s) h(s) d s \\
& x(0)=x_{0}
\end{aligned}
$$

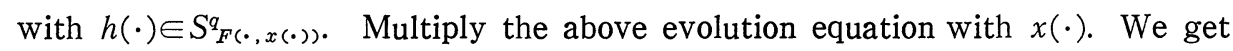

$$
\begin{aligned}
& \langle\dot{x}(t), x(t)\rangle+\langle A(t, x(t)), x(t)\rangle=\left(\int_{0}^{t} k(t-s) h(s) d s, x(t)\right) \text { a. e. } \\
& \Longrightarrow \frac{d}{d t}|x(t)|^{2}+2\langle A(t, x(t)), x(t)\rangle=2\left(\int_{0}^{t} k(t-s) h(s) d s, x(t)\right) \text { a. e. } \\
& \text { (see Tanabe [36], p. 151) } \\
& \Longrightarrow|x(t)|^{2}+2 c_{1} \int_{0}^{t}\|x(s)\|^{p} d s \leqq\left|x_{0}\right|^{2}+2 \int_{0}^{t} \int_{0}^{s}\|k\|_{\infty}|h(\tau)| d \tau\|x(s)\| d s .
\end{aligned}
$$

Using Cauchy's inequality with $\varepsilon>0$, we have

$$
2 \int_{0}^{t} \int_{0}^{s}\|k\|_{\infty}|h(\tau)| d \tau\|x(s)\| d s
$$




$$
\begin{aligned}
& \leqq \frac{2 \varepsilon^{p}}{p} \int_{0}^{t}\|x(s)\|^{p} d s+\frac{2}{q \varepsilon^{q}} \int_{0}^{t} \int_{0}^{s}\|k\|_{\infty}^{q}|h(\tau)|^{q} d \tau \\
& \leqq \frac{2 \varepsilon^{p}}{p} \int_{0}^{t}\|x(s)\|^{p} d s+\frac{2}{q \varepsilon^{q}} \int_{0}^{t} \int_{0}^{s}\|k\|_{\infty}^{q}\left(a(\tau)+b|x(\tau)|^{2 / q}\right)^{q} d \tau d s \\
& \leqq \frac{2 \varepsilon^{p}}{p} \int_{0}^{t}\|x(s)\|^{p} d s+\frac{2}{q \varepsilon^{q}} \int_{0}^{t} \int_{0}^{s}\|k\|_{\infty}^{q} 2^{q-1}\left(a(\tau)^{q}+b^{q}|x(\tau)|^{2}\right) d \tau d s .
\end{aligned}
$$

Let $\varepsilon^{p}=p c_{1}$. Then we have:

$$
\begin{aligned}
|x(t)|^{2} & \leqq \frac{2^{q}}{q(p c)^{q / p}}\|k\|_{\infty}^{q} \int_{0}^{t} \int_{0}^{s}\left(a(\tau)^{q}+b^{q}|x(\tau)|^{2}\right) d \tau d s \\
& =M \int_{0}^{t} \int_{0}^{s} a(\tau)^{q} d \tau d s+M b^{q} \int_{0}^{t} \int_{0}^{s}|x(\tau)|^{2} d \tau d s\left(M=\frac{2^{q}}{q\left(p c_{1}\right)^{q / p}}\|k\|_{\infty}^{q}\right)
\end{aligned}
$$

Invoking theorem 1 of Pachpatte [26], we get

$$
|x(t)| \leqq M_{1}, M_{1}>0 .
$$

Then we have:

$$
\begin{aligned}
2 c \int_{0}^{t}\|x(s)\|^{p} d s & \leqq\left|x_{0}\right|^{2}+2 \int_{0}^{t} \int_{0}^{s}\|k\|_{\infty}|h(\tau)| d \tau|x(s)| d s \\
& \leqq\left|x_{0}\right|^{2}+2 M_{1}\|k\|_{\infty} \int_{0}^{t} \int_{0}^{s}\left(a(\tau)+b M_{1}\right) d \tau \\
\Longrightarrow\|x\|_{L^{p}(X)} & \leqq M_{2}, M_{2}>0 .
\end{aligned}
$$

Next let $p(\cdot) \in L^{p}(X)$. We have :

$$
\begin{aligned}
\int_{0}^{t}\langle\dot{x}(s), p(s)\rangle d s & \leqq \int_{0}^{t}\|A(s, x(s))\|_{*} \cdot\|p(s)\| d s+\int_{0}^{t} \int_{0}^{s}\|k\|_{\infty}\|h(\tau)\|_{*} d \tau\|p(s)\| d s \\
& \leqq \int_{0}^{t}\left[c\left(1+\|x(s)\|^{p-1}\right)+\|k\|_{\infty} \int_{0}^{s}\|h(\tau)\|_{*} d \tau\right]\|p(s)\| d s
\end{aligned}
$$

Using Hölder's inequality, we get

$$
((\dot{x}, p))_{0} \leqq c(1+\|x(\cdot)\|)^{p-1}+\|k\|_{\infty}\|\eta(\cdot)\|_{L^{q}} \cdot\|p\|_{L} p_{(X)}
$$

where $\eta(s)=\int_{0}^{s}\|h(\tau)\|_{*} d \tau$ and $((\cdot, \cdot))_{0}$ denotes the duality brackets for the pair $\left(L^{p}(X), L^{q}\left(X^{*}\right)\right)$ (see Diestel-Uhl [12], p. 98). So we have

$$
((\dot{x}, p))_{0} \leqq\left(c r+c\|x\|_{L}^{p / q}(X)+\|k\|_{\infty}\|\eta\|_{\left.L^{q}\right) \cdot\|p\|_{L^{p}(X)}} .\right.
$$

Note that

$$
\|\eta\|_{q}^{q}=\int_{0}^{r} \eta(s)^{q} d s=\int_{0}^{r}\left(\int_{0}^{s}\|h(\tau)\|_{*} d \tau\right)^{q} d s \leqq \int_{0}^{r} \int_{0}^{s}\|h(\tau)\|_{*}^{q} d \tau d s \leqq \beta^{q} \int_{0}^{r} \int_{0}^{s}|h(\tau)|^{q} d \tau d s
$$


(where $\beta>0$ is such that $\|\cdot\|_{*} \leqq \beta|\cdot|$; it exists since $H \subset X^{*}$ continuouly). So

$$
\|\eta\|_{q}^{q} \leqq \int_{0}^{r} \int_{0}^{r} 2^{q-1}\left(a(\tau)^{q}+b^{q}|x(\tau)|^{2}\right) d \tau d s \leqq 2^{q-1} r\|a\|_{q}^{q}+2^{q-1} b^{q} M_{1}^{2} r^{2} \Longrightarrow\|\eta\|_{q} \leqq \bar{M}
$$

for some $\bar{M}>0$.

So finally we have $\|\dot{x}\|_{L^{q}\left(X^{*}\right)} \leqq M_{3}, M_{3}>0$.

Now define the following modification of the orientor field $F(t, x)$ :

$$
\hat{F}(t, x)=\left\{\begin{array}{lll}
F(t, x) & \text { if } & |x| \leqq M_{1} \\
F\left(t, \frac{M_{1} x}{|x|}\right) & \text { if } & |x|>M_{1}
\end{array}\right.
$$

Note that $\hat{F}(t, x)=F\left(t, p_{M_{1}}(x)\right)$, where $p_{M_{1}}(\cdot)$ is the $M_{1}$-radial retraction in $H$. Hence it is easy to see that $\hat{F}(t, x)$ has the same measurability and continuity properties as $F(t, x)$. Furthermore $|\hat{F}(t, x)|=\hat{a}(t)=a(t)+b M_{1}$ a. e., $\hat{a}(\cdot)$ $\in L^{q}$.

Set $V=\left\{h \in L^{q}(H):|h(t)| \leqq \hat{a}(t)\right.$ a. e. $\}$ and let $p: V \rightarrow W_{p q}(T)$ be defined by $p(h)(\cdot)=x(\cdot)$ where $x(\cdot) \in W_{p q}(T)$ is the unique solution of the evolution equation

$$
\dot{x}(t)+A(t, x(t))=\int_{0}^{t} k(t-s) h(s) d s \text { a. e., } x(0)=x_{0}
$$

(see Barbu [5] and Lions [22]).

Our claim is that this map $p(\cdot)$ is sequentially weakly continuous. To this end let $h_{n} \stackrel{w}{\rightarrow} h$ in $V$. Let $x_{n}(\cdot)=p\left(h_{n}\right)(\cdot) \in W_{p q}(T)$. From our a priori estimation in the beginning of the proof, we know that $\left\{x_{n}(\cdot)\right\}_{n \geq 1}$ is relatively sequentially weakly compact. So by passing to a subsequence if necessary, we may assume that $x_{n} \stackrel{w}{\rightarrow} x$ in $W_{p q}(T)$. We have:

$$
\dot{x}_{n}(t)+A\left(t, x_{n}(t)\right)=\int_{0}^{t} k(t-s) h_{n}(s) d s \text { a. e.. }
$$

Multiply the above equation with $x_{n}(t)-x(t)$. We get

$$
\left\langle\dot{x}_{n}(t), x_{n}(t)-x(t)\right\rangle+\left\langle A\left(t, x_{n}(t)\right), x_{n}(t)-x(t)\right\rangle=\left\langle u_{n}(t), x_{n}(t)-x(t)\right\rangle \text { a. e. }
$$

where $u_{n}(t)=\int_{0}^{t} k(t-s) h_{n}(s) d s$,

$$
\begin{gathered}
\Longrightarrow \int_{0}^{r}\left\langle\dot{x}_{n}(t), x_{n}(t)-x(t)\right\rangle d t+\int_{0}^{r}\left\langle A\left(t, x_{n}(t)\right), x_{n}(t)-x(t)\right\rangle d t \\
=\int_{0}^{r}\left\langle u_{n}(t), x_{n}(t)-x(t)\right\rangle d t
\end{gathered}
$$

Recall that (see Tanabe [36], p. 151) 


$$
\begin{aligned}
\left\langle\dot{x}_{n}(t)-\dot{x}(t), x_{n}(t)-x(t)\right\rangle=\frac{1}{2} & \frac{d}{d t}\left|x_{n}(t)-x(t)\right|^{2} \\
\Longrightarrow \int_{0}^{r}\left\langle\dot{x}_{n}(t), x_{n}(t)-x(t)\right\rangle d t= & \frac{1}{2}\left|x_{n}(r)-x(r)\right|^{2}-\frac{1}{2}\left|x_{n}(0)-x(0)\right|^{2} \\
& \quad+\int_{0}^{r}\left\langle\dot{x}(t), x_{n}(t)-x(t)\right\rangle d t
\end{aligned}
$$

So we get

$$
\begin{aligned}
\frac{1}{2}\left|x_{n}(r)-x(r)\right|^{2}+\int_{0}^{r}\langle\dot{x}(t), & \left.x_{n}(t)-x(r)\right\rangle d t+\int_{0}^{r}\left\langle A\left(t, x_{n}(t)\right), x_{n}(t)-x(t)\right\rangle d t \\
= & \int_{0}^{r}\left\langle u_{n}(t), x_{n}(t)-x(t)\right\rangle d t
\end{aligned}
$$

Since $W_{p q}(T) \hookrightarrow L^{p}(H)$ compactly (see section 2) and $W_{p q}(T) \hookrightarrow C(T, H)$, by passing to a subsequence if necessary, we may assume that $x_{n}(t) \stackrel{s}{\rightarrow} x(t)$ in $H$ for all $t \in T$. So we have:

$$
\begin{aligned}
& \frac{1}{2}\left|x_{n}(r)-x(r)\right|^{2} \longrightarrow 0 \\
& \int_{0}^{b}\left\langle\dot{x}(t), x_{n}(t)-x(t)\right\rangle d t \longrightarrow 0
\end{aligned}
$$

Also note that $u_{n}(t) \stackrel{w}{\rightarrow} u(t)=\int_{0}^{t} k(t-s) h(s) d s$ in $H$ for all $t \in T$. So we have:

$$
\begin{aligned}
& \int_{0}^{b}\left\langle u_{n}(t), x_{n}(t)-x(t)\right\rangle d t \longrightarrow 0 \\
& \Longrightarrow \overline{\lim }\left(\left(\hat{A} x_{n}, x_{n}-x\right)\right)_{0}=0\left(\left(\tilde{A} x_{n}\right)(t)=A\left(t, x_{n}(t)\right)\right)
\end{aligned}
$$

Note that because of hypothesis $H(A)(2)$ and by passing to a subsequence if necessary, we may assume that $\hat{A} x_{n} \stackrel{w}{\rightarrow} v$ in $L^{q}\left(X^{*}\right)$. Using this and relation (1) above, we deduce that

$$
\varlimsup
$$

But using hypothesis $H(A)$, it is easy to check that $\hat{A}(\cdot)$ is hemicontinuous, monotone, everywhere defined on $L^{p}(X)$, hence is pseudomonotone (see Browder [7]) and so it has property $(M)$. Therefore $\hat{A} x=v$, i. e. $\hat{A} x_{n} \stackrel{w}{\rightarrow} \hat{A} x$ in $L^{q}\left(X^{*}\right)$. So

$$
\begin{aligned}
& \left(\left(\dot{x}_{n}, p\right)\right)_{0}+\left(\left(\hat{A} x_{n}, p\right)\right)_{0}=\left(\left(u_{n}, p\right)\right)_{0} \\
& \quad \longrightarrow((\dot{x}, p))_{0}+((\hat{A} x, p))_{0}=((u, p))_{0} \text { as } n \rightarrow \infty
\end{aligned}
$$

Since $p \in L^{p}(X)$ was arbitrary, we conclude that 


$$
\begin{aligned}
& \dot{x}(t)+A(t, x(t))=u(t) \quad \text { a. e. } \\
& \Longrightarrow \dot{x}(t)+A(t, x(t))=\int_{0}^{t} k(t-s) h(s) d s \text { a. e., } x(0)=x_{0} \\
& \Longrightarrow x(\cdot)=p(h)(\cdot) \\
& \Longrightarrow p(\cdot) \text { is sequentially weakly continuous as claimed. }
\end{aligned}
$$

Next let $R: V \rightarrow P_{f c}(V)$ be defined by $R(h)=S_{F(\cdot, p(h)(\cdot)) \text {. Our claim now is }}^{1}$ that $R(\cdot)$ is u. s. c. on $V$ with the weak topology. Note that $V$ is bounded in the reflexive Banach space $L^{q}(H)$, hence it is sequentially weakly compact. So we only need to show that $G r R$ is sequentially weakly closed in $V \times V$ (see for example Klein-Thompson [17]). So let $\left\{\left(h_{n}, f_{n}\right)\right\}_{n \geqq 1} \subseteq G r R$ and assume $\left(h_{n}, f_{n}\right)$ $\stackrel{w x w}{\longrightarrow}(h, f)$ in $V \times V$. Then from what we proved above, we have $p\left(h_{n}\right) \stackrel{w}{\rightarrow} p(h)$ in $W_{p q}(T) \Rightarrow p\left(h_{n}\right)(t) \stackrel{s}{\rightarrow} p(h)(t)$ in $H$ for all $t \in T\left(\right.$ recall $W_{p q}(T) \hookrightarrow L^{p}(H)$ compactly and $W_{p q}(T) \hookrightarrow C(T, H)$ ). Because of hypothesis $H(F)(2)$ and since $\hat{F}(\cdot, \cdot)$ has the same continuity properties as $F(\cdot, \cdot)$ we have $w-\overline{\lim } \hat{F}\left(t, p\left(h_{n}\right)(t)\right) \subseteq \hat{F}(t, p(h)(t))$. So invoking theorem 4.3 of [31], we get

$$
\begin{aligned}
& f \in w-\overline{\lim } R\left(h_{n}\right)=w-\overline{\lim } S_{\hat{F}\left(\cdot, p\left(h_{n}\right)(\cdot)\right) \subseteq S_{\hat{F}(\cdot, p(h)(\cdot))}^{1}=R(h)}^{1} \\
& \Longrightarrow(h, f) \in G r R \text { i. e. } R(\cdot) \text { is u. s. c. as claimed. }
\end{aligned}
$$

Apply the Kakutani-KyFan fixed point theorem to get $h \in V$ s. t. $h \in R(h)$. Then clearly $p(h)(\cdot)=x(\cdot)$ solves $(*)$ with the orientor field $\hat{F}(t, x)$. Then by the same estimation as in the beginning of the proof, we have $|x(t)| \leqq M_{1} \Rightarrow$ $\hat{F}(t, x(t))=F(t, x(t)) \Rightarrow x(\cdot) \in W_{p q}(T)$ is the desired solution.

Q.E. D.

An interesting consequence of the above proof, is the following property of the solution set of $(*)$.

THEOREM 3.2. If hypotheses $H(A), H(F)$ and $H(k)$ hold, then the solution set of $\left(^{*}\right)$ is a nonempty, weakly compact subset of $W_{p q}(T)$.

Proof. Let $P\left(x_{0}\right)$ be the solution set of $\left(^{*}\right)$. We have already seen in the proof of theorem 3.1 that $P\left(x_{0}\right) \subseteq p(V)$ where $V \cong L^{q}(H)$ and $p: L^{q}(H) \rightarrow W_{p q}(T)$ are as in the proof of theorem 3.1. Since $p(\cdot)$ is sequentially weakly continuous and $V \subseteq L^{q}(H)$ is weakly compact, we have that $p(V)$ is weakly compact in $W_{p q}(T)$. So it suffices to show that $P\left(x_{0}\right)$ is sequentially weakly closed in $W_{p q}(T)$. Hence let $\left\{x_{n}\right\}_{n \geqq 1} \subseteq P\left(x_{0}\right), x_{n} \stackrel{w}{\rightarrow} x$ in $W_{p q}(T)$. We have

$$
\dot{x}_{n}(t)+A\left(t, x_{n}(t)\right)=h_{n}(t) \text { a. e., } x_{n}(0)=x_{0}
$$

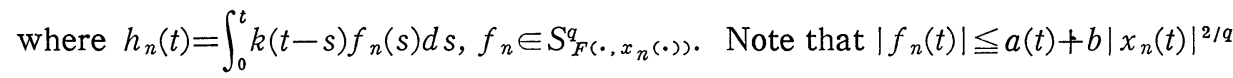
$\leqq a(t)+b M_{1}^{2 / q} \Rightarrow\left|f_{n}(t)\right|^{q} \leqq 2^{q-1} a(t)^{q}+2^{q-1} b^{q} M_{1}^{2} \Rightarrow\left\|f_{n}\right\|_{L^{q}(H)} \leqq 2^{q-1}\|a\|_{q}^{q}+2^{q-1} b^{q} M_{1}^{2} r=$ 
$M_{4}$. (recall the a priori estimates obtained in the proof of theorem 3.1). So $\left\{f_{n}\right\}_{n \geqq 1}$ is bounded in $L^{q}(H)$, hence relatively sequentially weakly compact. Thus by passing to a subsequence if necessary, we may assume that $f_{n} \stackrel{w}{\rightarrow} f$ in $L^{q}(H)$. Then invoking theorem 3.1 of [31], we have $f(t) \in \overline{\operatorname{conv}} w-\overline{\lim }\left\{f_{n}(t)\right\}_{n \geqq 1}$ $\cong \overline{\operatorname{conv}} w-\overline{\lim } F\left(t, x_{n}(t)\right)$ a. e.. But as before since $W_{p q}(T) \hookrightarrow L^{p}(H)$ compactly and $W_{p q}(T) \hookrightarrow C(T, H)$, by passing to a subsequence if necessary, we may assume that $x_{n}(t) \stackrel{s}{\rightarrow} x(t)$ in $H$ for all $t \in T$. So because of hypothesis $H(F)(2)$, we have that $w-\overline{\lim } F\left(t, x_{n}(t)\right) \subseteq F(t, x(t))$ (see Delahaye-Denel [11]) $\Rightarrow f(t) \in F(t, x(t))$ a. e. $\Rightarrow$

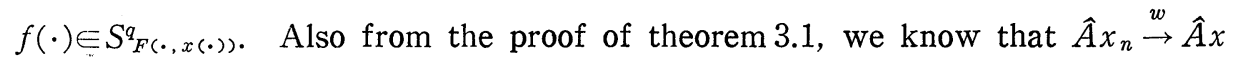
in $L^{q}\left(X^{*}\right)$, while $h_{n} \stackrel{w}{\rightarrow} h$ in $L^{q}(H)$ with $h(t)=\int_{0}^{t} k(t-s) f(s) d s$ and $\dot{x}_{n} \stackrel{w}{\rightarrow} \dot{x}$ in $L^{q}\left(X^{*}\right)$. So by passing to the limit in (1) we get

$$
\dot{x}(t)+A(t, x(t))=h(t) \text { a. e., } x(0)=x_{0}
$$

From (2) we deduce that $x \in P\left(x_{0}\right) \Rightarrow P\left(x_{0}\right)$ is indeed nonempty, weakly compact in $W_{p q}^{r}(T)$.

Q.E. D.

Remark. Since $W_{p q}(T) \subset L^{p}(H)$ compactly, we get that $P\left(x_{0}\right)$ is compact in $L^{p}(H)$. Also, if $p=q=2$ and $X$ is a separable Hilbert space, then from Nagy [24], we know that $W(T) \hookrightarrow C\left(T, H^{\prime}\right)$ compactly. So, in that case, $P\left(x_{0}\right)$ is compact $C(T, H)$.

Next we will consider the case where the orientor field $F(t, x)$ is nonconvex valued. We will need the following hypothesis on $F(t, x)$.

$H(F)_{1}: \quad F: T \times H \rightarrow P_{f}(H)$ is a multifunction s. t.

(1) $F(\cdot, \cdot)$ is graph measurable,

(2) $F(t, \cdot)$ is 1 . s. c.

(3) $|F(t, x)| \leqq a(t)+b|x|^{2 / q}$ a. e. with $a(\cdot) \in L^{q}, b>0$.

THEOREM 3.3. If hypotheses $H(A), H(F)_{1}$ and $H^{\prime}(k)$ hold, then (*) admits a solution.

Proof. Let $B=p(V)$, where $V \subseteq L^{q}\left(H^{\prime}\right)$ and $p: L^{q}(H) \rightarrow W_{p q}(T)$ are as in the proof of theorem 3.1. We know that $B$ is a weakly compact subset of $W_{p q}(T)$ and by the Krein-Smulian theorem so is $\hat{B}=\overline{\operatorname{conv}} B$ (see Diestel-Uhl [12], p. 51).

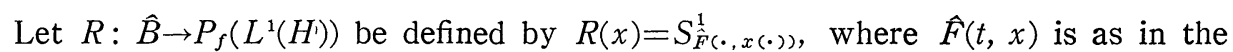
proof of theorem 3.1. Combining hypothesis $H(F)_{1}(2)$ with theorem 4.1 of [31], we get that $R(\cdot)$ is 1 . s. c.. So we can apply the selection theorem of BressanColombo [6] and get $r: \hat{B} \rightarrow L^{1}(H)$ continuous from $\hat{B}$ with the relative weak $W_{p q}(T)$-topology (which is metrizable; see Dunford-Schwartz [13], p. 434) into $L^{1}(H)$ with the strong topology s. t. $r(x) \in R(x)$ for all $x \in \hat{B}$. Let $\eta(x)(\cdot) \in$ $W_{p q}(T)$ be the unique solution of the evolution equation $\dot{y}(t)+A(t, y(t))=$ 
$\int_{0}^{t} k(t-s) r(x)(s) d s, y(0)=x_{0}$ (see Barbu [5] and Lions [22]). Then $\eta(x)(\cdot) \in \hat{B}$ and so $\eta: \hat{B} \rightarrow \hat{B}$. We claim that $\eta(\cdot)$ is continuous for the relative weak $W_{p q}(T)$-topology on $\hat{B}$. Recalling that this topology is metrizable, we can use sequences to check the continuity of $\eta(\cdot)$. So let $x_{n} \rightarrow x$ in $\hat{B}$. Then $r\left(x_{n}\right) \stackrel{s}{\rightarrow}$ $r(x)$ in $L^{1}(H)$. Also $\left\{\eta\left(x_{n}\right)\right\}_{n \geqq 1}$ is bounded in $W_{p q}(T)$ and so we may assume that $\eta\left(x_{n}\right) \stackrel{w}{\rightarrow} z$ in $W_{p q}(T)$. Then working as before, in the limit as $n \rightarrow \infty$ we will get that

$$
\begin{aligned}
& \dot{z}(t)+A(t, z(t))=\int_{0}^{t} k(t-s) r(x)(s) d s, x(0)=x_{0} \\
& \Longrightarrow z=\eta(x) \\
& \Longrightarrow \eta(\cdot) \text { is indeed continuous as claimed. }
\end{aligned}
$$

Applying the Schauder-Tichonov fixed point theorem, we get $x \in \hat{B}$ s. $t$. $\eta(x)=x$. This is the solution of $\left(^{*}\right)$ with the orientor field $\hat{F}(t, x)$. An estimation as in the beginning of the proof of theorem 3.1 gives us that $|x(t)| \leqq M_{1} \Rightarrow$ $\hat{F}(t, x(t))=F(t, x(t)) \Rightarrow x(\cdot)$ solves $(*)$.

Q. E. D.

\section{Sensitivity analysis}

In this section we examine the dependence of the solutions of $(*)$ on the data of the problem; i. e. on the operator $A$, on the orientor field $F(t, x)$ and on the initial condition.

So consider the following sequence of integrodifferential evolution inclusions :

$$
\left\{\begin{array}{l}
\dot{x}_{n}(t)+A_{n}(t) x_{n}(t) \in \int_{0}^{t} k(t-s) F_{n}\left(s, x_{n}(s)\right) d s \text { a.e. } \\
x_{n}(0)=x_{0}^{n}
\end{array}\right\}(* *)_{n}
$$

and the limit integrodifferential evolution inclusion

$$
\left\{\begin{array}{l}
\dot{x}(t)+A(t) x(t) \in \int_{0}^{t} k(t-s) F(s, x(s)) d s \text { a. e. } \\
x(0)=x_{0}
\end{array}\right\}(* *)
$$

We will need a mode of convergence for the linear operators $\left\{A_{n}(t)\right\}_{n \geqq 1} \subseteq$ $\mathcal{L}\left(X, X^{*}\right)$ and $\left\{d / d t+\hat{A}_{n}\right\}_{n \geqq 1}$. Following Zhikov-Kozlov-Oleinik [40], we make the following definitions.

Definition I. A sequence of linear operators $A_{n}: X \rightarrow X^{*} n \geqq 1, G$-converges to a linear operator $A: X \rightarrow X^{*}$ as $n \rightarrow \infty$, if the operators $A_{n}^{-1}, A^{-1}: X^{*} \rightarrow X$ are defined and for any $x^{*} \in X^{*}, A_{n}^{-1} x^{*} \stackrel{w}{\rightarrow} A^{-1} x^{*}$ in $X$ (strongly in $H$, since $X \hookrightarrow H$ compactly). 
Definition II. A sequence of operators $P_{n}: W(T) \rightarrow L^{2}\left(X^{*}\right) \times H, n \geqq 1$, " $P G$ converges" to an operator $P: W(T) \rightarrow L^{2}\left(X^{*}\right) \times H^{*}$ as $n \rightarrow \infty$, if the operators $P_{n}^{-1}$, $P^{-1}: L^{2}\left(X^{*}\right) \times H \rightarrow W(T)$ are defined and for any $\left(g, x_{0}\right) \in L^{2}\left(X^{*}\right) \times H, P_{n}^{-1}\left(g, x_{0}\right)$ $\stackrel{w}{\rightarrow} P^{-1}\left(g, x_{0}\right)$ in $W(T)$ as $n \rightarrow \infty\left(P_{n}^{-1}\left(g, x_{0}\right)(t) \stackrel{s}{\rightarrow} P^{-1}\left(g, x_{0}\right)(t)\right.$ as $n \rightarrow \infty$ in $H$ for all $t \in T)$.

Here the operator $P_{n}(\cdot)$ will be defined by $x \in W(T) \rightarrow\left(\dot{x}(\cdot)+A_{n}(\cdot) x(\cdot), x_{0}\right) \in$ $L^{2}\left(X^{*}\right) \times H$ (the dynamics of the approximating problems) and the operator $P(\cdot)$ will be defined by $x(\cdot) \in W(T) \rightarrow\left(\dot{x}(\cdot)+A(\cdot) x(\cdot), x_{0}\right)$ (the dynamics of the limit problem). So definition I refers to the convergence of the solutions of a sequence of elliptic problems, while definition II refers to the convergence of the solutions of a sequence of parabolic problems.

We will need the following hypotheses on the data of problems $(* *)_{n}$ and $\left(^{* *}\right)$

$H(A)_{1}: A_{n}, A: T \rightarrow \mathcal{L}\left(X, X^{*}\right)$ are operators s. t.

(1) $t \rightarrow A_{n}(t) x, A(t) x$ are measurable,

(2) $x \rightarrow A_{n}(t) x, A(t) x$ are continuous, monotone,

(3) $\left\|A_{n}(t) x\right\|_{*},\|A(t) x\|_{*} \leqq c\|x\|$ a. e. with $c>0$,

(4) $\left\langle A_{n}(t) x, x\right\rangle,\langle A(t) x, x\rangle \geqq c_{1}\|x\|^{2}$ a. e.

(5) $A_{n}(t) \stackrel{G}{\rightarrow} A(t)$ a. e. and given $\varepsilon>0$ there exists $\delta(\varepsilon)>0$ s. t. for all $n \geqq 1$ $\sup _{0 \leqq t \leqq b}\left\|A_{n}(t+\tau)-A_{n}(t)\right\|_{*}<\varepsilon$ provided $\tau \in(0, \delta)$.

$H(F)_{2}: F_{n}, F: T \times H \rightarrow P_{f c}(H)$ are multifunctions s. t.

(1) $F_{n}(\cdot, \cdot), F(\cdot, \cdot)$ are graph measurable,

(2) $h\left(F_{n}(t, x), F_{n}(t, y)\right) \leqq l(t)|x-y|$ a. e. with $l(\cdot) \in L_{+}^{1}$

(3) $\left|F_{n}(t, x)\right| \leqq a(t)+b|x|$ a. e. with $a(\cdot) \in L_{+}^{2}, b>0$,

(4) $F_{n}(t, x) \stackrel{n}{\rightarrow} F(t, x)$.

Denote the solution set of $(* *)_{n}$ by $P_{n}$ and the solution set of $(* *)$ by $P$.

THEOREM 4.1. If hypotheses $H(A)_{1}, H(F)_{2}, H(k)$ hold and $x_{0}^{n} \stackrel{s}{\rightarrow} x_{0}$ in $H$, then $\overline{\lim } P_{n} \leqq P$ in $C(T, H)$.

Proof. Let $x_{n}(\cdot) \in P_{n}, n \geqq 1$ and $x_{n} \rightarrow x$ in $C(T, H)$. By definition we have

$$
\left\{\begin{array}{l}
\dot{x}_{n}(t)+A_{n}(t) x_{n}(t)=\int_{0}^{t} k(t-s) h_{n}(s) d s \text { a. e. } \\
x_{n}(0)=x_{0}^{n}
\end{array}\right\}(1)
$$

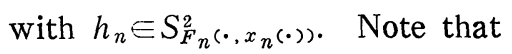

$$
\begin{aligned}
& h\left(F_{n}\left(t, x_{n}(t)\right), F(t, x(t))\right) \leqq h\left(F_{n}\left(t, x_{n}(t)\right), F_{n}(t, x(t))\right)+h\left(F_{n}(t, x(t)), F(t, x(t))\right) \\
& \Longrightarrow h\left(S_{F_{n}\left(\cdot, x_{n}(\cdot)\right)}^{1}, S_{F(\cdot, x(\cdot))}^{1}\right) \leqq h\left(S_{F\left(\cdot, x_{n}(\cdot)\right)}^{1}, S_{F_{n}(\cdot, x(\cdot))}^{1}\right)+h\left(S_{\left.F_{n}(\cdot, x(\cdot))\right)}^{1}\right),
\end{aligned}
$$$$
\left.S_{F(\cdot, x(\cdot))}^{1}\right) \leqq\|l\|_{1}\left\|x_{n}-x\right\|_{C(T, H)}+h\left(S_{F_{n}(\cdot, x(\cdot))}^{1}, S_{F(\cdot, x(\cdot))}^{1}\right) \rightarrow 0
$$ 
(see [31], theorem 4.5).

Note that because of hypothesis $H(F)_{2}(3),\left\{h_{n}\right\}_{n \geqq 1}$ is bounded in $L^{2}(H)$ and so by passing to a subsequence if neccssary, we may assume that $h_{n} \stackrel{w}{\rightarrow} h$ in $L^{2}(H)$. Then for every $u \in L^{2}(H)=L^{2}(H)^{*}$ we have

$$
\begin{aligned}
& \left(h_{n}, u\right)_{L^{2}(H)} \leqq \sup \left\{(f, u)_{L^{2}(H)}: f \in S_{F_{n}\left(\cdot, x_{n}(\cdot)\right)}^{2}=S_{F_{n}\left(\cdot, x_{n}(\cdot)\right)}^{1}\right\}=\sigma_{\left.S_{F_{n}(\cdot, x}^{1}(\cdot)\right)}(u) \\
& \Longrightarrow(h, u)_{L^{2}(H)} \leqq \sigma_{S_{F(\cdot, x(\cdot))}^{1}}(u) \quad \text { for every } u \in L^{2}(H)
\end{aligned}
$$

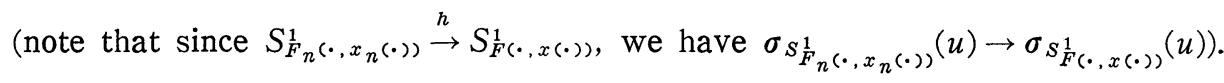

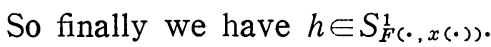

Fix $y(\cdot) \in W_{r}(T)=\{z(\cdot) \in W(T): z(r)=0\}$ and set

$$
-\dot{y}(t)+A(t) * y(t)=g(t)
$$

Multiply equation (1) with $y_{n}(\cdot)$, equation (2) with $x_{n}(\cdot)$ and then subtract the new equation (2) from the new equation (1). Then integrate over $T=[0, r]$. We get:

$$
\begin{aligned}
& \int_{0}^{r}\left\langle\dot{x}_{n}(t), y_{n}(t)\right\rangle d t+\int_{0}^{r}\left\langle\dot{y}_{n}(t), x_{n}(t)\right\rangle d t+\int_{0}^{r}\left\langle A_{n}(t) x_{n}(t), y_{n}(t)\right\rangle d t \\
& \quad-\int_{0}^{r}\left\langle A_{n}(t)^{*} y_{n}(t), x_{n}(t)\right\rangle d t=\int_{0}^{r}\left\langle v_{n}(t), y_{n}(t)\right\rangle d t-\int_{0}^{r}\left\langle g(t), x_{n}(t)\right\rangle d t
\end{aligned}
$$

where $v_{n}(t)=\int_{0}^{t} k(t-s) h_{n}(s) d s$. Invoking lemma 5.5.1, p. 151 of Tanabe [36], we can perform integration by parts on the second integral in the left hand side of (3). Doing that we get

$$
\begin{aligned}
\int_{0}^{r}\left\langle\dot{y}_{n}(t), x_{n}(t)\right\rangle d t & =\left(y_{n}(r), x_{n}(r)\right)-\left(y_{n}(0), x_{n}(0)\right)-\int_{0}^{r}\left\langle y_{n}(t), \dot{x}_{n}(t)\right\rangle d t \\
& =-\left(y_{n}(0), x_{0}^{n}\right)-\int_{0}^{r}\left\langle y_{n}(t), \dot{x}_{n}(t)\right\rangle d t
\end{aligned}
$$

Substituting (4) in (3), we get

$$
-\left(y_{n}(0), x_{0}^{n}\right)=\int_{0}^{r}\left(v_{n}(t), y_{n}(t)\right) d t-\int_{0}^{r}\left\langle g(t), x_{n}(t)\right\rangle d t
$$

From theorem 10 of Zhikov-Kozlov-Oleinik [40], we know that $\mathscr{P}_{n}=$ $\frac{d}{d t}+\hat{A}_{n} \stackrel{P G}{\rightarrow} \mathscr{P}=\frac{d}{d t}+\hat{A}$ and so theorem 7 of the same paper tells us that $\mathcal{Q}_{n}^{*} \stackrel{P G}{\longrightarrow} \mathcal{Q}^{*}$. Then invoking lemma 3 of Zhikov-Kozlov-Oleinik [40], we get that $y_{n} \stackrel{s}{\rightarrow} y$ in $C(T, H)$. So since $x_{n} \stackrel{w}{\rightarrow} x$ in $W(T)$, in the limit as $n \rightarrow \infty$, we get from equation (5) 


$$
\begin{aligned}
&-\left(y(0), x_{0}\right)=\int_{0}^{r}(v(t), y(t)) d t-\int_{0}^{r}\langle g(t), x(t)\rangle d t,\left(v(t)=\int_{0}^{t} k(t-s) h(s) d s\right) \\
&=\int_{0}^{r}(v(t), y(t)) d t-\int_{0}^{r}\left\langle-\dot{y}(t)+A(t)^{*} y(t), x(t)\right\rangle d t \\
&=\int_{0}^{r}(v(t), y(t)) d t-\left\langle y(0), x_{0}\right)-\int_{0}^{r}\langle y(t), \dot{x}(t)\rangle d t-\int_{0}^{b}\langle y(t), A(t) x(t)\rangle d t \\
& \Longrightarrow \int_{0}^{r}\langle\dot{x}(t)+A(t) x(t), y(t)\rangle d t=\int_{0}^{r}\langle v(t), y(t)\rangle d t \\
& \Longrightarrow(\dot{x}+A x, y)_{L^{2}(X), L^{2}\left(X^{*}\right)}=(v, y)_{L^{2}(X), L^{2}\left(X^{*}\right)}
\end{aligned}
$$

Since $y \in W_{r}(T)$ was arbitrary and $W_{r}(T)$ is dense in $L^{2}(X)$ (see ZhikovKozlov-Oleinik [40]), we conclude that

$$
\dot{x}(t)+A(t) x(t)=v(t) \text { a. e., } x(0)=x_{0}
$$

with $v(t)=\int_{0}^{t} k(t-s) h(s) d s, h(\cdot) \in S_{F(\cdot, x(\cdot))}^{1}$. Hence $x(\cdot) \in P$ and thus we have shown that $\overline{\lim } P_{n} \subseteq P$.

Q. E. D.

Remarks. (1) If $X$ is a separable Hilbert space, then $w-\overline{\lim } P_{n} \subseteq P$. This follows easily from theorem 4.1 and that fact in this case $W(T) \subseteq C(T, H)$ compactly (see Nagy [24]). Recall that $\overline{\lim } P_{n} \leqq w-\overline{\lim } P_{n}=\{x(\cdot) \in C(T, H): x=w$ $\left.\lim x_{n_{k}}, x_{n_{k}} \in P_{n_{k}}\right\}$.

(2) It will be interesting to know if and when we have $P_{n} \stackrel{K}{\rightarrow} P$ in $C(T, H)$. Recall that $K$ denotes the Kuratowski convergence of sets (i. e. $\underline{\lim } P_{n}=\{x \in$ $\left.C(T, H): \lim d\left(x, P_{n}\right)=0\right\}=P=\overline{\lim } P_{n}=\left\{x(\cdot) \in C(T, H): \underline{\lim } d\left(x, P_{n}\right)=0\right\}$; see Kuratowski [19]). The difficulty here is the "memory" feature of our evolution inclusion. Namely, because of the integral term, the velocity doesn't only depend on the instantaneous values of the state, but also on the past ones.

\section{Random version}

In this section we examine a random version of the integrodifferential evolution inclusion $(*)$. So now all the data depend on a random parameter $\omega$ belonging in a complete probability space $(\Omega, \Sigma, \mu)$.

So the integrodifferential evolution inclusion under consideration is now the following :

$$
\left\{\begin{array}{l}
\dot{x}(\omega, t)+A(\omega, t, x(\omega, t)) \in \int_{0}^{t} k(\omega, t-s) F(\omega, s, x(\omega, s)) d s \text { a. e. on } T, \omega \in \Omega \\
x(\omega, 0)=x_{0}(\omega)
\end{array}\right\}(*)_{\omega}
$$

By a random solution of $(*)_{\omega}$, we understand a measurable stochastic process $x: \Omega \times T \rightarrow X$ s. t. for every $\omega \in \Omega, x(\omega, \cdot) \in W_{p q}(T)$ and $x(\omega, \cdot)$ solves $(*)_{\omega}$. 
We will need the following hypotheses on the data

$H(A)_{2}: A: \Omega \times T \times X \rightarrow X^{*}$ is an operator s. t.

(1) $(\omega, t) \rightarrow A(\omega, t, x)$ is measurable,

(2) $x \rightarrow A(\omega, t, x)$ is hemicontinuous, monotone,

(3) $\|A(\omega, t, x)\|_{*} \leqq c(\omega)\left(1+\|x\|^{p-1}\right)$ a. e. for all $\omega \in \Omega, c(\omega)>0, c(\cdot)$ is measurable, $p \geqq 2$,

(4) $\langle A(\omega, t, x), x\rangle \geqq c_{1}(\omega)\|x\|^{P}$ a. e., $c_{1}(\omega)>0, c_{1}(\cdot)$ is measurable.

$H(F)_{3}: \quad F: \Omega \times T \times H \rightarrow P_{f}(H)$ is a multifunction s.t.

(1) $F(\cdot, \cdot, \cdot)$ is measurable,

(2) $F(\omega, t, \cdot)$ is sequentially closed in $H \times H_{w}$ for $F(\cdot, \cdot, \cdot)$ being $P_{f c}(H)$ valued or $F(\omega, t, \cdot)$ is 1 . s. c. for $F(\cdot, \cdot, \cdot)$ being simply $P_{f}(H)$-valued,

(3) $|F(\omega, t, x)| \leqq a(\omega, t)+b(\omega)|x|^{2 / q}$ a. e. for all $\omega \in \Omega, a(\omega, \cdot) \in L_{+}^{q}, a(\cdot, \cdot)$ is measurable,

$H(k)_{1}: \quad k(\omega, \cdot) \in L^{\infty}(T, \mathcal{L}(H))$ and for all $h \in H(\omega, t) \rightarrow k(\omega, t) h$ is measurable.

THEOREM 5.1. If hypotheses $H(A)_{2}, H(F)_{3}, H(k)_{1}$ hold and $x_{0}: \Omega \rightarrow H$ is measurable, then $(*)_{\omega}$ admits a random solution.

Proof. Let $S: \Omega \rightarrow 2^{W} q^{(T)}$ be defined by

$$
\begin{gathered}
S(\omega)=\left\{x \in W_{p q}(T): \dot{x}(t)+A(\omega, t, x(t))=\int_{0}^{t} k(\omega, t-s) h(s) d s \text { a. e., } x(0)=x_{0}(\omega)\right. \\
\left.h \in S_{F(\omega, \cdot, x(\cdot))}^{q}=S_{F(\omega, \cdot x(\cdot))}^{1}\right\} .
\end{gathered}
$$

From theorem 3.1 (convex case) or theorem 3.3 (nonconvex case), we have that $S(\omega) \neq \varnothing$ for all $\omega \in \Omega$. Then

$$
\begin{aligned}
G r S=\{(\omega, x) \in \Omega & \times W_{p q}(T): \int_{0}^{r}\langle\dot{x}(t), u(t)\rangle d t+\int_{0}^{r}\langle A(\omega, t, x(t)), u(t)\rangle d t \\
& =\int_{0}^{r}\left(\int_{0}^{t} k(\omega, t-s) h(s) d s, u(t)\right) d t, u \in L^{p}(X), x(0)=x_{0}(\omega) \\
& \text { and } \left.\int_{0}^{r} d_{H}(h(t), F(\omega, t, x(t))) d t=0\right\}
\end{aligned}
$$

where $d_{H}$ denotes the distance function in $H$. Since $X$ is separable, $L^{p}(X)$ is separable too. So let $\left\{u_{n}\right\}_{n \geqq 1} \subseteq L^{p}(X)$ be a countable dense subset. We have:

$$
\begin{gathered}
G r S=\bigcap_{n \geqq 1}\left\{(\omega, x) \in \Omega \times W_{p q}(T): \int_{0}^{r}\left\langle\dot{x}(t), u_{n}(t)\right\rangle d t+\int_{0}^{r}\left\langle A(\omega, t, x(t)), u_{n}(t)\right\rangle d t\right. \\
\quad=\int_{0}^{r}\left(\int_{0}^{t} k(\omega, t-s) h(s) d s, u_{n}(t)\right) d t, x(0)=x_{0}(\omega) \\
\text { and } \left.\int_{0}^{r} d_{H}(h(t), F(\omega, t, x(t))) d t=0\right\} .
\end{gathered}
$$


Recall that $x \rightarrow \dot{x}$ is a continuous map from $W_{p q}(T)$ into $L^{q}\left(X^{*}\right)$. Also note that $(\omega, x) \rightarrow \int_{0}^{r}\left\langle A(\omega, t, x(t)), u_{n}(t)\right\rangle d t$ is a Caratheodory function, i. e. measurable in $\omega$ and continuous in $x$; hence it is jointly measurable. Also from Fubini's theorem we have that $\omega \rightarrow \int_{0}^{r}\left(\int_{0}^{t} k(\omega, t-s) h(s) d s, u_{n}(t)\right) d t n \geqq 1$ is measurable. Finally let $i: W_{p q}(T) \rightarrow C(T, H)$ be the continuous embedding map. Using the measurability hypothesis on $F(\cdot, \cdot, \cdot)$ ) (see hypothesis $H(F)_{3}(1)$ ), we see that $(\omega, t, x, z) \rightarrow d_{H}\left(z, F\left(\omega, t, e_{t}(i(x))\right)\right.$ (here $e_{t}(\cdot)$ is the $t$-evaluation map) is measurable in $(\omega, t, x)$ and of course continuous in $z$, thus it is jointly measurable. Hence $(\omega, t, x) \rightarrow d_{H}(h(t), F(\omega, t, x(t)))$ is measurable from $\Omega \times T \times W_{p q}(T, H)$ into $\boldsymbol{R}_{+} \Rightarrow$ $\phi:(\omega, x) \rightarrow \int_{0}^{b} d_{H}(h(t), F(\omega, t, x(t))) d t$ is measurable. Furthermore the map $\phi:$ $(\omega, x) \rightarrow e_{0}(i(x))-x_{0}(\omega)$ is Caratheodory, hence jointly measurable. So finally

$$
\begin{aligned}
G r S=\bigcap_{n \geq 1}\{(\omega, x) & \in \Omega \times W_{p q}(T): \int_{0}^{r}\left\langle\dot{x}(t), u_{n}(t)\right\rangle d t+\int_{0}^{r}\left\langle A(\omega, t, x(t)), u_{n}(t)\right\rangle d t \\
& \left.=\int_{0}^{r}\left(\int_{0}^{t} k(\omega, t-s) h(s) d s, u_{n}(t)\right) d t, \phi(\omega, x)=0, \phi(\omega, x)=0\right\} \\
& \in \Sigma \times B\left(W_{p q}(T)\right) .
\end{aligned}
$$

Apply Aumann's selection theorem (see Wagner [38]) to find $s: \Omega \rightarrow W_{p q}(T)$ measurable s.t. $s(\omega) \in S(\omega)$ for all $\omega \in \Omega$. Then invoking theorem 17, p. 198 of Dunford-Schwartz [13], we conclude that $x(\omega, t)=s(\omega)(t)$ is the desired random $\begin{array}{ll}\text { solution of }(*)_{\omega} \text {. } & \text { Q. E. D. }\end{array}$

Remark. The interesting feature of the above existence result is that it covers also the nonconvex case. Most works in the literature, even for simple differential inclusions in $\boldsymbol{R}^{n}$, assume convexity and continuity of the random orientor field (see for example Chuong [9], Nowak [25] and Papageorgiou [27], [29]) or single valuedness of it (see for example Deimling [10], KravvaritisPapageorgiou [18], Ladde-Lakshmikantham [20] and Tsokos-Padgett [37]).

\section{Optimal control}

In this section we consider applications to optimal control.

So consider the following infinite dimensional optimal control problem with "memory".

$$
\left\{\begin{array}{ll}
J(x, & u)=\int_{0}^{r} L(t, x(t), u(t)) d t \rightarrow \inf =m \\
\text { s. t. } & \dot{x}(t)+A(t, x(t))=\int_{0}^{t} k((t-s) f(s, x(s)) u(s) d s . \text { a. e. } \\
& x(0)=x_{0} \in H \\
& u(t) \in U(t, x(t)) \text { a. e., } u(\cdot) \text { is measurable }
\end{array} \text { (***) }^{* *}\right.
$$


We will need the following hypotheses on the data. Here $Y$ is a separable reflexive Banach space, modelling the control space.

$H(f): f: T \times H \rightarrow \mathcal{L}(Y, H)$ is a map s.t.

(1) $t \rightarrow f(t, x) u$ is measurable for all $(x, u) \in H \times Y$ (i. e. $f(\cdot, x)$ is measurable for the strong operator topology),

(2) $x \rightarrow f(t, x)^{*} h$ is continuous for all $(t, h) \in T \times H$ (i. e. the adjoint of $f(t, x)$ is continuous in $x$ in the strong operator topology),

(3) $\|f(t, x)\|_{\mathcal{L}(Y, H)} \leqq a(t)+b|x|^{2 / q}$ a. e., with $a(\cdot) \in L_{+}^{9}, b>0$.

$H(U): \quad U: T \times H \rightarrow P_{f c}(Y)$ is a multifunction s. t.

(1) $U(\cdot, \cdot)$ is graph measurable,

(2) $x \rightarrow U(t, x)$ has a graph which is sequentially closed in $H \times Y_{w}$ (here $Y_{w}$ denotes the space $Y$ with its weak topology),

(3) $|U(t, x)| \leqq M$ for all $(t, x) \in T \times H$.

$H(L): \quad L: T \times H \times Y \rightarrow \overline{\boldsymbol{R}}=\boldsymbol{R} \cup\{+\infty\}$ is an integrand s. t.

(1) $L(\cdot, \cdot, \cdot)$ is measurable,

(2) $L(t, \cdot, \cdot)$ is 1 . s. c. on $H \times Y$ and convex in $u \in Y$,

(3) $\phi(t)-M(|x|+\|u\|) \leqq L(t, x, u)$ a. e. with $\phi(\cdot) \in L^{1}, M>0$.

Because of the feedback form of our control constraints and since our cost integrand is $\bar{R}$-valued, we also need the following feasibility type hypothesis:

$H_{0}$ : There exists an admissible "state-control" pair $(x, u) \in W_{p q}(T) \times L^{1}(Y)$ for $(* * *)$ s. t. $J(x, u)<\infty$.

THEOREM 6.1. If hypotheses $H(A), H(f), H(k), H(U), H(L)$ and $H_{0}$ hold, then (***) has a nonempty, sequentially weakly compact in $W_{p q}(T) \times L^{1}(Y)$ set of optimal "state-control" pairs.

Proof. Let $F: T \times H \rightarrow P_{f c}(H)$ be defined by $F(t, x)=f(t, x) U(t, x)$. We claim that $F(\cdot, \cdot)$ satisfies hypothesis $H(F)$. First note that:

$$
\begin{aligned}
G r F & =\{(t, x, y) \in T \times H \times H: y \in F(t, x)\} \\
& =\{(t, x, y) \in T \times H \times H: y=f(t, x) u, u \in U(t, x)\} \\
& =\operatorname{proj}_{r \times H \times H}\left[(t, x, y, u) \in T \times H \times H \times B_{M}(0): y=f(t, x) u,(t, x, u) \in G r U\right]
\end{aligned}
$$

where $B_{M}(0)=\{v \in Y:\|v\| \leqq M\}$.

Note that $L=\left\{(t, x, y, u) \in T \times H \times H \times B_{M}(0): y=f(t, x) u,(t, x, u) \in G r U\right\}$ $\in B(T) \times B(H) \times B(H) \times B\left(B_{M}(0)\right)$. Also $B_{M}(0)_{w}$ (i. e. $B_{M}(0)$ with the relative weak topology) is compact metrizable (see Dunford-Schwartz [13], p. 434) and $B\left(B_{M}(0)_{w}\right)=B\left(Y_{w}\right) \cap B_{M}(0)=B(Y) \cap B_{M}(0)=B\left(B_{M}(0)\right)$ (see Edgar [14]). So invoking the Arsenin-Novikov theorem (see Levin [21]), we get that $\operatorname{proj}_{T \times H \times H} L \in$ $B(T) \times B(H) \times B(H)$. 
Next we will show that $\operatorname{Gr} F(t, \cdot)$ is sequentially closed in $H \times H_{w}$. So let $\left(x_{n}, y_{n}\right) \in G r F(t, \cdot)$ and assume that $\left(x_{n}, y_{n}\right) \stackrel{s x w}{\longrightarrow}(x, y)$. We have $y_{n}=f\left(t, x_{n}\right) u_{n}$ with $u_{n} \in U\left(t, x_{n}\right)$. Since $\left\|u_{n}\right\| \leqq M, n \geqq 1$, by passing to a subsequence if necessary, we may assume that $u_{n} \rightarrow u$ and because of hypotheses $H(U)(2)$ and (3) we have that $u \in w-\overline{\lim } U\left(t, x_{n}\right) \leqq U(t, x)$. Also for all $h \in H$ we have

$$
\left(y_{n}, h\right)=\left(f\left(t, x_{n}\right) u_{n}, h\right)=\left(u_{n}, f\left(t, x_{n}\right)^{*} h\right)
$$

But by hypothesis $H(f)(2), x \rightarrow f(t, x)^{*} h$ is continuous. So

$$
\begin{aligned}
\left(u_{n}, f\left(t, x_{n}\right)^{*} h\right)_{Y, Y^{*}} \longrightarrow\left(u, f(t, x)^{*} h\right)_{Y, Y^{*}} & \\
& \Longrightarrow(f(t, x) u, h)=(y, h) \quad \text { for all } h \in H, \\
& \Longrightarrow y=f(t, x) u \quad \text { with } \quad u \in U(t, x), \\
& \Longrightarrow(x, y) \in G r F,
\end{aligned}
$$

Finally note that $|F(t, x)| \leqq\|f(t, x)\|_{\mathcal{L}(Y, H)} M \leqq a(t) M+b M|x|^{2 / q}$. So we have satisfied hypothesis $H(F)$.

Now note that every trajectory of $(* * *)$ also solves the integrodifferential evolution inclusion $\dot{x}(t)+A(t, x(t)) \in \int_{0}^{t} k(t-s) F(s, x(s)) d s, x(0)=x_{0}$ and the latter has a solution set that is weakly compact in $W_{p q}(T)$ (see theorem 3.2). So to prove our theorem, we need to show that the set of optimal pairs is nonempty and sequentially weakly closed in $W_{p q}(T) \times L^{1}(Y)$.

To this end let $\left\{\left(x_{n}, u_{n}\right)\right\}_{n \geq 1}$ be a minimizing sequence of admissible pairs, i. e. $J\left(x_{n}, u_{n}\right) \downarrow m$. By passing to an appropriate subsequence, we may assume that $x_{n} \stackrel{w}{\rightarrow} x$ in $W_{p q}(T)$ and $u_{n} \stackrel{w}{\rightarrow} u$ in $L^{1}(Y)$. Invoking theorem 2.1 of Balder [4] we get

$$
\int_{0}^{r} L(t, x(t), u(t)) d t \leqq m=\lim J\left(x_{n}, u_{n}\right) .
$$

Also $\dot{x}_{n} \stackrel{w}{\rightarrow} \dot{x}$ and $\hat{A} x_{n} \stackrel{w}{\rightarrow} \hat{A} x$ in $L^{q}\left(X^{*}\right)$ (see the proof of theorem 3.1) and since $W_{p q}(T) \hookrightarrow L^{p}(H)$ compactly and $W_{p q}(T) \hookrightarrow C(T, H)$, we may assume that $x_{n}(t) \stackrel{s}{\rightarrow} x(t)$ in $H$ for all $t \in T$ and so by using hypothesis $H(f)(2)$, we have $\int_{0}^{t} k(t-s) f\left(s, x_{n}(t)\right) u_{n}(s) d s \stackrel{w}{\rightarrow} \int_{0}^{t} k(t-s) f(s, x(s)) u(s) d s$ in $H$. Thus for every $p \in$ $L^{p}(X)$ we have

$$
\begin{aligned}
& \left(\left(\dot{x}_{n}, p\right)\right)_{0}+\left(\left(\hat{A} x_{n}, p\right)\right)_{0}=\left(\left(v_{n}, p\right)\right)_{0}\left(v_{n}(t)=\int_{0}^{t} k(t-s) f\left(s, x_{n}(s)\right) u_{n}(s) d s\right) \\
& \longrightarrow((\dot{x}, p))_{0}+((\hat{A} x, p))_{0}=((v, p))_{0}\left(v(t)=\int_{0}^{t} k(t-s) f(s, x(s)) u(s) d s\right),
\end{aligned}
$$




$$
\begin{aligned}
\Longrightarrow & \dot{x}(t)+A(t, x(t))=\int_{0}^{t} k(t-s) f(s, x(s)) u(s) d s \text { a. e. } \\
& x(0)=x_{0} \\
& u(t) \in U(t, x(t)) \text { a. e., } u(\cdot) \text { is measurable } \\
\Longrightarrow & (x, u) \text { is an admissible "state-control" pair for }\left(^{* * *}\right) \\
\Longrightarrow & J(x, u)=m \text { i. e. }(x, u) \text { is optimal. }
\end{aligned}
$$

So the set of optimal pairs is nonempty. Furthermore it is clear from the above proof that it is sequentially weakly closed in $W_{p q}(T) \times L^{1}(Y)$. Thus it is sequentially weakly compact in $W_{p q}(T) \times L^{1}(Y)$.

Now we turn our attention to the variational stability of the optimal control problems. So consider the following sequence of problems:

$$
\left\{\begin{array} { c } 
{ J _ { n } ( x , u ) = \int _ { 0 } ^ { r } L _ { n } ( t , x ( t ) , u ( t ) ) d t \rightarrow \operatorname { i n f } = m _ { n } } \\
{ \text { s.t. } \dot { x } ( t ) + A _ { n } ( t ) x ( t ) = \int _ { 0 } ^ { t } k ( t - s ) f _ { n } ( s , x ( s ) ) u ( s ) d s \text { a. e. } } \\
{ x ( 0 ) = x _ { 0 } ^ { n } } \\
{ u ( t ) \in U _ { n } ( t ) \text { a. e., } u ( \cdot ) \text { is measurable } }
\end{array} \left({ }^{* * *)_{n},}\right.\right.
$$

and the limit problem

$$
\left\{\begin{array}{c}
J(x, u)=\int_{0}^{b} L(t, x(t), u(t)) d t \rightarrow \inf =m \\
\text { s. t. } \dot{x}(t)+A(t) x(t)=\int_{0}^{t} k(t-s) f(s, x(s)) u(s) d s \text { a. e. } \\
x(0)=x \\
u(t) \in U(t) \text { a. e., } u(\cdot) \text { is measurable }
\end{array}\right\}(* * *),
$$

Recall that if $Z$ is a Banach space and $\left\{C_{n}, C\right\}_{n \geq 1} \subseteq 2^{Z} \backslash\{\varnothing\}$, we define $\underline{\lim } C_{n}=\left\{z \in Z: \lim d\left(z, C_{n}\right)=0\right\}=\left\{z \in Z: z=s-\lim z_{n}, z_{n} \in C_{n}, n \geqq 1\right\}, \overline{\lim } C_{n}=\{z \in Z:$ $\left.\underline{\lim } d\left(z, C_{n}\right)=0\right\}=\left\{z \in Z: z=s-\lim z_{n_{k}}, z_{n_{k}} \in C_{n_{k}}, n_{1}<n_{2}<\cdots<n_{k}<\cdots\right\}$ and $w-\overline{\lim } C_{n}$ $=\left\{z \in Z: z=w-\lim z_{n_{k}}, z_{n_{k}} \in C_{n_{k}}, n_{1}<n_{2}<\cdots<\cdots n_{k}<\cdots\right\}$. We say that the $C_{n}$ 's converge to $C$ in the Kuratowski sense (denoted by $C_{n} \stackrel{K}{\rightarrow} C$ ) if and only if $\underline{\lim } C_{n}=C=\varlimsup{ }_{\lim } C_{n}$ (see Kuratowski [19]). We also say that the $C_{n}$ 's converge to $C$ in the Kuratowski-Mosco sense (denoted by $C_{n} \stackrel{K-M}{\longrightarrow} C$ ) if and only if $\underline{\lim } C_{n}=C=w$ - $\overline{\lim } C_{n}$ (see Mosco [23]). Note that we always have $\underline{\lim } C_{n} \subseteq \overline{\lim } C_{n}$ $\subseteq w-\overline{\lim } C_{n}$. If $\left\{f_{n}, f\right\}_{n \geq 1} \subseteq \overline{\boldsymbol{R}}^{z}$, then we say that the $f_{n}$ 's epi-converge to $f$ (denoted by $f_{n} \stackrel{r}{\rightarrow} f$ ) if and only if epif $\stackrel{K-M}{\longrightarrow}$ epif. Recall that epif $=\{(z, \lambda) \in$ $Z \times \boldsymbol{R}: f(z) \leqq \lambda\}$ (similarly for epif $_{n}$ ). This mode of convergence is in general 
different from pointwise convergence and is useful in the sensitivity analysis of variational problems. For more details we refer to Attouch [3] and Mosco [23].

We will need the following hypotheses on the data of our problems:

$H(f)_{1}: f_{n}, f: T \times H \rightarrow \mathcal{L}(Y, H)$ are maps s. t.

(1) $t \rightarrow f_{n}(t, x) u$ are measurable for all $(x, u) \in H \times Y$,

(2) $\left|f_{n}(t, x)^{*} u-f_{n}(t, \dot{y})^{*} u\right| \leqq l_{L}(t)|x-y|$ a. e. with $l_{L}(\cdot) \in L_{+}^{1},\|u\| \leqq L, x$, $y \in H$,

(3) $\left\|f_{n}(t, x)\right\|_{\mathcal{L}(Y, H)} \leqq a(t)+b|x|$ a. e. with $a(\cdot) \in L_{+}^{2}, b>0$,

(4) $f_{n}(t, x) \stackrel{s o}{\rightarrow} f(t, x)$ a. e., where so denotes the strong operator topology.

$H(U)_{1}: U_{n}, U: T \rightarrow P_{f c}(Y)$ are multifunctions s. t.

(1) $U_{n}(\cdot)$ are measurable,

(2) $\left|U_{n}(t)\right| \leqq M$ a. e.,

(3) $U_{n}(t) \stackrel{K}{\rightarrow} U(t)$ a. e..

$H(L)_{1}: L_{n}, L: T \times H \times Y \rightarrow \boldsymbol{R}$ are integrands s. t.

(1) $t \rightarrow L_{n}(t, x, u)$ are measurable,

(2) $(x, u) \rightarrow L_{n}(t, x, u), L(t, x, u)$ are bontinuous, convex,

(3) $\left|L_{n}(t, x, u)\right| \leqq \phi(t)+b\left(|x|^{2}+\|u\|^{2}\right)$ a. e. with $\phi(\cdot) \in L_{+}^{2}, b>0$,

(4) $L_{n}(t, \cdot, \cdot) \stackrel{r}{\rightarrow} L(t, \cdot, \cdot)$ a. e..

THEOREM 6.2. Jf hypotheses $H(A)_{1}, H(f)_{1}, H(k), H(U)_{1}, H(L)_{1}$ hold and $x_{0}^{n}$ $\stackrel{s}{\rightarrow} x_{0}$ in $H$, then $m_{n} \rightarrow m$.

Proof. Let $(x, u)$ be an admissible "state-control" pair s. t. $J(x, u)=m$. It existence is guaranteed by theorem 6.1. Note that for every $v \in L^{1}(Y)$ we have:

$$
\begin{aligned}
d_{S_{U_{n}}^{1}}(v) & =\inf _{w \in S^{1}}\|v-w\|_{1}=\inf _{w \in S_{U_{n}}^{1}} \int_{0}^{b}\|v(t)-w(t)\| d t \\
& =\int_{0}^{b} \inf _{h \in U_{n}(t)}\|v(t)-h\| d t=\int_{0}^{b} d_{U(t)}(v(t)) d t
\end{aligned}
$$

and similarly $d_{S_{U}^{1}}(v)=\int_{0}^{b} d_{U(t)}(v(t)) d t$. Because by hypothesis $H(U)_{1} U_{n}(t) \stackrel{K}{\rightarrow} U(t)$ a. e. we have $d_{U_{n}(t)}(v(t)) \rightarrow d_{U(t)}(v(t))$ a. e. and so by the dominated convergence theorem we have $d_{S_{U_{n}}^{1}}(v) \rightarrow d_{S_{U}^{1}}(v)$.

Let $u_{n} \in S_{U_{n}}^{1}$ s.t. $d_{S_{U_{n}}^{1}}(u)=\left\|u-u_{n}\right\|_{L^{1}(Y)}$. Such an element exists since by proposition 3.1 of [30], $S_{U_{n}}^{1}$ is weakly compact in $L^{1}(Y)$. Then $d_{S_{U_{n}}^{1}}(u)=$ $\left\|u-u_{n}\right\|_{L^{1}(Y)} \rightarrow d_{S_{U}^{1}}(u)=0$. Let $x_{n}(\cdot) \in W(T)$ be the unique trajectory generated by $u_{n}(\cdot)$. By passing to a subsequence if necessary, we may assume that $x_{n} \stackrel{w}{\rightarrow} \hat{x}$ in $W(T)$ and so $x_{n} \stackrel{s}{\rightarrow} \hat{x}$ in $L^{2}(H)$. Working with the auxiliary function $y(\cdot) \in$ $W_{r}(T)$ as in the proof of theorem 4.1, we can show that $\hat{x}(\cdot)$ is the unique trajectory generated by the admissible control $u(\cdot)$. Also from theorem 3.1 of 
Salvadori [34], we know that $J_{n} \stackrel{\tau}{\rightarrow} J$ and so from Mosco [23] (see also Salvadori [34]), we know that we can find $y_{n} \stackrel{s}{\rightarrow} x$ in $L^{2}(H)$ and $v_{n} \stackrel{s}{\rightarrow} u$ in $L^{1}(Y)$ s.t. $\overline{\lim } J_{n}\left(y_{n}, v_{n}\right) \leqq J(x, u)=m$. From Rockafellar [33], we know that $\left\{J_{n}(\cdot, \cdot)\right\}_{n \geqq 1}$ is locally equi-Lipschitz. Thus for some $\hat{k}>0$ we have

$$
\begin{aligned}
& \left|J_{n}\left(x_{n}, u_{n}\right)-J_{n}\left(y_{n}, v_{n}\right)\right| \leqq \hat{k}\left[\left\|x_{n}-y_{n}\right\|_{L^{2}(H)}+\left\|u_{n}-v_{n}\right\|_{L^{2}(Y)}\right] \rightarrow 0 \\
& \Longrightarrow\left|J_{n}\left(x_{n}, u_{n}\right)-J_{n}\left(y_{n}, v_{n}\right)\right| \rightarrow 0
\end{aligned}
$$

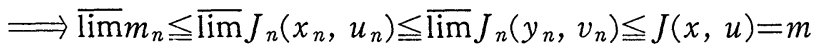

$$
\begin{aligned}
& \Longrightarrow \overline{\lim } m_{n} \leqq m
\end{aligned}
$$

Next let $\left\{\left(x_{n}, u_{n}\right)\right\}_{n \geq 1}$ be admissible "state-control" pairs for $(* * *)_{n}^{\prime} n \geqq 1$ s. t. $J_{n}\left(x_{n}, u_{n}\right)=m_{n}$. Again their existence is guaranteed by theorem 6.1. By passing to a subsequence if necessary, we may assume that $x_{n} \stackrel{w}{\rightarrow} x$ in $W(T)$ and $\underset{n}{u_{n}} \stackrel{w}{\rightarrow} u$ in $L^{1}(Y)$. Clearly $u \in S_{U}^{1}$. Also, once again as in the proof of theorem 4.1, through the auxiliary function $y \in W_{r}(T)$, we can show that $(x, u)$ is admissible for $(* * *)^{\prime}$ (note that

So

$$
\begin{aligned}
& \left\|f_{n}\left(t, x_{n}(t)\right) * v-f(t, x(t)) * v\right\|_{\mathcal{L}(Y, H)} \leqq\left\|f_{n}\left(t, x_{n}(t)\right) * v-f_{n}(t, x(t)) * v\right\|_{\mathcal{L}(Y, H)} \\
& \quad+\left\|f_{n}(t, x(t)) * v-f(t, x(t)) * v\right\|_{\mathcal{L}(Y, H)} \leqq l_{L}(t)\left|x_{n}(t)-x(t)\right|+\| f_{n}(t, x(t)) * v \\
& \quad-f(t, x(t)) * v \|_{\mathcal{L}(Y, H) \rightarrow 0 \text { a. e. for }\|v\| \leqq L .}
\end{aligned}
$$

$$
\int_{0}^{t} k(t-s) f_{n}\left(s, x_{n}(s)\right) u_{n}(s) d s \stackrel{w}{\longrightarrow} \int_{0}^{t} k(t-s) f(s, x(s)) u(s) d s
$$

in $H$ ). Then since $J_{n} \stackrel{r}{\rightarrow} J$, from the properties of the epigraphical convergence (see Mosco [23] and Salvadori [34]), we have

$$
m \leqq J(x, u) \leqq \underline{\lim } m_{n}=\underline{\lim } J_{n}\left(x_{n}, u_{n}\right) \longrightarrow m \leqq \underline{\lim } m_{n}
$$

From (1) and (2) above, we conclude that

$$
m_{n} \longrightarrow m \text {. Q.E. D. }
$$

\section{Examples}

In this last section of the paper we work out in detail two examples that ilustrate the applicability of our work.

(a) In the first example we consider a distributed parameter optimal control problem, governed by a nonlinear parabolic integrodifferential equation. $\partial Z=\Gamma$.

So let $T=[0, r]$ and let $Z$ be a bounded domain in $\boldsymbol{R}^{n}$ with smooth boundary

The problem under consideration is the following: 


$$
\left\{\begin{array}{c}
J(x, u)=\int_{0}^{b} \int_{Z} L(t, z, x(t, z), u(t, z)) d z d t \longrightarrow \inf =m \\
\text { s. t. } \frac{\partial n}{\partial t}+\sum_{|\alpha| \leqq m}(-1)^{|\alpha|} D^{\alpha} A_{\alpha}\left(t, z, \eta(x(t, z))=\int_{0}^{t} k(t-s) f(s, z, x(t, z))\right. \\
u(s, z) d s \text { on } T \times Z \\
\left.D^{\beta} x\right|_{T \times \Gamma}=0|\beta| \leqq m-1, x(0, z)=x_{0}(z), \int_{Z}|u(t, z)|^{2} d z \\
\leqq \int_{Z} \psi(t, z, x(t, z))^{2} d z
\end{array}\right\}(* * * *)
$$

Here $\alpha=\left(\alpha_{1}, \cdots, \alpha_{n}\right)$ is a multi-index, $D^{\alpha}=D_{1}^{\alpha_{1}} \ldots D_{n}^{\alpha} n$ is the elementary partial differential operator with $D_{i}=\frac{\partial}{\partial z_{\imath}}$. Also $|\alpha|=\sum_{i=1}^{n}\left|\alpha_{\imath}\right|$ is the "length" of the multi-index $\alpha$ and $\eta(x(z))=\left\{D^{r} x(z):|\gamma| \leqq m\right\}$.

We take $X=H_{0}^{m}(Z), H=L^{2}(Z)$ and $X^{*}=H^{-m}(Z)$. It is well known that this is a Gelfand triple of spaces, with all embeddings being compact (SobolevKondrachov embedding theorem).

We will need the following hypotheses on the data of $(* * *)$ :

$H(A)_{3}: \quad A_{\alpha}: T \times Z \times \boldsymbol{R}^{n(m)} \rightarrow \boldsymbol{R}\left(n(m)=\frac{(n+m) !}{n ! m !}\right)$ are maps s.t.

(1) $(t, z) \rightarrow A_{\alpha}(t, z, \eta)$ are measurable,

(2) $\eta \rightarrow A_{\alpha}(t, z, \eta)$ is continuous,

(3) $\left|A_{\alpha}(t, z, \eta)\right| \leqq a(t, z)+b\|\eta\|$ a. e. with $a(\cdot, \cdot) \in L_{+}^{2}(T \times Z), b>0$,

(4) $\sum_{|\alpha| \geqq m}\left(A_{\alpha}\left(t, z, \eta_{1}\right)-A_{\alpha}\left(t, z, \eta_{2}\right)\right)\left(\eta_{1 \alpha}-\eta_{2 \alpha}\right) \geqq 0$,

(5) $c\|\eta\|^{2} \leqq \sum_{|\alpha| \leqq m} A_{\alpha}(t, z, \eta) \eta_{\alpha}$ with $c>0$.

$H(f)_{2}: f: T \times Z \times \boldsymbol{R} \rightarrow \boldsymbol{R}$ is a map s. t.

(1) $(t, z) \rightarrow f(t, z, x)$ is measurable,

(2) $x \rightarrow f(t, z, x)$ is continuous

(3) $|f(t, z, x)| \leqq M_{1}(t, z)$ a. e. with $M_{1}(\cdot, \cdot) \in L^{\infty}(T \times Z)$.

$H(L)_{2}: \quad L: T \times Z \times \boldsymbol{R} \times \boldsymbol{R} \rightarrow \overline{\boldsymbol{R}}$ is an integrand s.t.

(1) $L(\cdot, \cdot, \cdot, \cdot)$ is measurable

(2) $(x, u) \rightarrow L(t, z, x, u)$ is 1 . s. c. and convex in $u$,

(3) $\phi(t, z)-M(z)(|x|+|u|) \leqq L(t, z, x, u)$ a. e. with $\phi(\cdot, \cdot) \in L^{1}(T \times Z)$, $M(\cdot) \in L_{+}^{1}(Z)$.

$H(\phi): \quad \psi: T \times Z \times \boldsymbol{R} \rightarrow \boldsymbol{R}$ is a map s. t.

(1) $(t, z) \rightarrow \psi(t, z, x)$ is measurable,

(2) $x \rightarrow \psi(t, z, x)$ is continuous,

(3) $|\psi(t, z, x)| \leqq M$. by

Let $a: T \times H_{0}^{m}(Z) \times H_{0}^{m}(Z) \rightarrow \boldsymbol{R}$ be the time dependent Dirichlet form defined 


$$
a(t, x, y)=\sum_{|\alpha| \leqq m} \int_{Z} A_{\alpha}(t, z, \eta(x(z))) D y^{\alpha}(z) d z, x, y \in H_{0}^{m}(Z) .
$$

Using the Cauchy-Schwartz and Minkowski inequalities, we can show that

$$
|a(t, x, y)| \leqq\left(\hat{a}(t)+b\|x\|_{H_{0}^{m}(Z)}^{2}\right)\|y\|_{H_{0}^{m}(Z)}
$$

with $\hat{a}(t)=\|a(t, \cdot)\|_{L^{2}(Z)}$. So we can define a generally nonlinear operator $A(t)$ : $H_{0}^{m}(Z) \rightarrow H^{-m}(Z)$ by

$$
a(t, x, y)=\langle A(t, x), y\rangle .
$$

From Krasnoselski's theorem, we know that the Nemitsky (superposition) operators corresponding to the maps $A_{\alpha}(t, \cdot, \eta(x(\cdot)))$ are continuous from $H_{0}^{m}(Z)$ into $L^{2}(Z)$. Using that, it is easy to check that $A(t, \cdot)$ is hemicontinuous. Also clearly from Fubini's theorem, we have that $t \rightarrow A(t, x)$ is measurable.

Next note that

$$
\begin{aligned}
\langle A(t, x)-A(t, y), x-y\rangle= & \int_{Z|\alpha| \leqq m} \sum_{\infty}\left(A_{\alpha}(t, z, \eta(x(z)))-A_{\alpha}(t, z, \eta(y(z)))\right) \\
& \times\left(D^{\alpha} x(z)-D^{\alpha} y(z)\right) d z \geqq 0
\end{aligned}
$$

$\Longrightarrow A(t, \cdot)$ is monotone.

Finally from hypothesis $H(A)_{3}(5)$ we have that

$$
\begin{aligned}
& c \sum_{|\alpha| \leqq m}\left|D^{\alpha} x(z)\right|^{2} \leqq \sum_{|\alpha| \leqq m} A_{\alpha}(t, z, \eta(x(z))) D^{\alpha} x(z) \text { a. e. } \\
& \Longrightarrow c\|x\|_{H_{0}^{m}(z)}^{2} \leqq\langle A(t, x), x\rangle \text { a. e. }
\end{aligned}
$$

Therefore we have satisfied hypothesis $H(A)$.

Next let $\hat{f}: T \times L^{2}(Z) \rightarrow \mathcal{L}\left(L^{2}(Z)\right)$ be defined by

$$
(\hat{f}(t, x) u)(z)=f(t, z, x(z)) u(z) .
$$

Because of hypothesis $H(f)_{2}$ we see that $\hat{f}(\cdot, \cdot)$ is well defined and satisfies hypothesis $H(f)$.

Let $\hat{L}: T \times L^{2}(Z) \times L^{2}(Z) \rightarrow \overline{\boldsymbol{R}}$ be defined by

$$
\hat{L}(t, x, u)=\int_{z} L(t, z, x(z), u(z)) d z .
$$

Since $L(\cdot, \cdot, \cdot, \cdot)$ is a normal integrand (i. e. measurable in all variables and 1. s. c. in $(x, u)$; see for example Salvadori [34]), we can find Caratheodory integrands $L_{n}(t, z, x, u)$ s. t. $L_{n} \uparrow L$ and $\phi(t, z)-M(z)(|x|+|u|) \leqq L_{n}(t, z, x, u) \leqq$ $n$. Set $\hat{L}_{n}(t, x, u)=\int_{z} L_{n}(t, z, x(z), u(z)) d z$. Clearly $\hat{L}_{n}(\cdot, \cdot, \cdot \cdot)$ is Caratheodory, hence jointly measurable and by the Monotone Convergence Theorem we have $\hat{L}_{n} \uparrow \hat{L} \Rightarrow \hat{L}$ is jointly measurable. Also $\hat{L}(t, \cdot, \cdot)$ is 1 . s. c. and convex in $u$. 
So we have satisfied $H(L)$.

Finally let $\hat{k}: T \rightarrow \mathcal{L}\left(L^{2}(Z)\right)$ be defined by $k(t) I$ and let

$$
U(t, x)=\left\{u \in L^{2}(Z):\|u\|_{L^{2}(Z)}^{2} \hat{\phi}(t, x)^{2}\right\}
$$

where $\hat{\phi}(t, x)=\int_{z} \psi(t, z, x(z)) d z$. Note that because of hypothesis $H(\phi) \hat{\psi}(\cdot, \cdot)$ is a Caratheodory function (hence jointly measurable) and so $U(t, x)$ satisfies hypothesis $H(U)$.

Now rewrite $(* * * *)$ as the following equivalent abstract optimal control problem:

$$
\left\{\begin{array}{c}
\hat{J}(x, u)=\int_{0}^{r} \hat{L}(t, x(t), u(t)) d t \rightarrow \inf =m \\
\text { s.t. } \dot{x}(t)+A(t, x(t))=\int_{0}^{t} \hat{k}(t-s) \hat{f}(s, x(s)) u(s) d s \text { a. e. } \\
x(0)=x_{0}(\cdot) \\
u(t) \in U(t, x(t)) \text { a. e., } u(\cdot) \text { is measurable }
\end{array}\right\}(* * * *)
$$

This has the form of problem (***). So invoking theorem 6.1 , we have:

THEOREM 7.1. If hypotheses $H(A)_{3}, H(f)_{2}, H(k), H(L)_{2}, H(\phi)$ hold and $x_{0}(\cdot)$ $\in L^{2}(Z)$, then $(* * *)$ admits an optimal solution.

(b) This second example deals with the variational stability of a class of parabolic optimal control problems.

So consider the following sequence of optimal control problem:

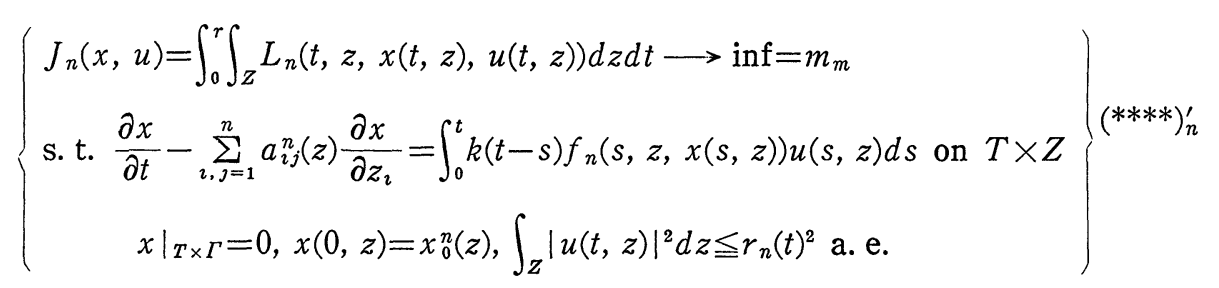

and the limit problem

$$
\left\{\begin{array}{l}
J(x, u)=\int_{0}^{r} \int_{z} L(t, z, x(t, z), u(t, z)) d z d t \longrightarrow \inf =m \\
\text { s. t. } \frac{\partial x}{\partial t}-\sum_{\imath, j=1}^{n} a_{\imath j}(z) \frac{\partial x}{\partial z_{\imath}}=\int_{0}^{t} k(t-s) f(s, z, x(s, z)) u(s, z) d s \text { on } T \times Z \\
\left.x\right|_{T \times \Gamma}=0, x(0, z)=x_{0}(z), \int_{Z}|u(t, z)|^{2} d z \leqq r(t)^{2} \text { a. e. }
\end{array}\right\}(* * * *)^{\prime}
$$

Here $X=H_{0}^{1}(Z), H=L^{2}(Z)$ and $X^{*}=H^{-1}(Z)$. We will need the following hypotheses on the data of the above problems. 
$H(A)_{4}: \quad a_{\imath \jmath}=a_{j i}$, there exist $m_{1}, m_{2}>0$ s.t. $m_{1} \leqq a_{2 j}^{n}(z) \leqq m_{1}$ and $1 / a_{11}^{n} \stackrel{w^{*}}{\rightarrow} 1 / a_{11}$ $\frac{a_{i 1}^{n}}{a_{11}^{n}} \stackrel{w^{*}}{\longrightarrow} \frac{a_{i 1}}{a_{11}}, a_{\imath j}^{n}-\frac{a_{i 1}^{n} a_{\imath \jmath}^{n}}{a_{11}^{n}} \stackrel{w^{*}}{\longrightarrow} a_{\imath j}-\frac{a_{i 1} a_{\imath \jmath}}{a_{11}}(i, j \neq 1)$ in $L^{\infty}(Z)$.

$H(f)_{3}: f_{n}, f: T \times Z \times \boldsymbol{R} \rightarrow \boldsymbol{R}$ are functions satisfying $H(f)_{2}$ and $f_{n}(t, z, x) \rightarrow$ $f(t, z, x)$

$H(L)_{3}: \quad L_{n}, L: T \times Z \times \boldsymbol{R} \times \boldsymbol{R} \rightarrow \boldsymbol{R}$ are Caratheodory integrand convex in $(x, u)$ $\in \boldsymbol{R} \times \boldsymbol{R}$,

$\left|L_{n}(t, z, x, u)\right|,|L(t, z, x, u)| \leqq \phi_{1}(t, z)+b\left(|x|^{2}+|u|^{2}\right)$ a. e. with $\phi_{1}(\cdot, \cdot)$

$\in L^{2}(T \times Z), b>0$ and $L_{n}(t, \cdot, \cdot, \cdot) \stackrel{\tau}{\longrightarrow} L(t, \cdot, \cdot, \cdot)$ a. e.

$H(r): \quad\left|r_{n}(t)\right| \leqq M$ a. e. $n \geqq 1$ and $r_{n}(t) \rightarrow r(t)$ a. e..

Let $A_{n}, A: H_{0}^{1}(Z) \rightarrow H^{-1}(Z)$ be the bounded linear operators defined by

$$
\left\langle A_{n}, x, y\right\rangle=a_{n}(x, y)=\int_{z} \sum_{\imath, \jmath=1}^{n} a_{\imath j}^{n}(z) \frac{\partial x}{\partial z_{\imath}} \frac{\partial x}{\partial z_{\jmath}} d z
$$

and

$$
\langle A x, y\rangle=a(x, y)=\int_{Z} \sum_{\imath, \jmath=1}^{n} a_{\imath j}(z) \frac{\partial x}{\partial z_{\imath}} \frac{\partial x}{\partial z_{\jmath}} d z .
$$

Then $A_{n}, A \in \mathcal{L}\left(X, X^{*}\right)$ and because of hypothesis $H(A)_{4}$ and Tartar's theorem (see Sokolowski [35]), we have $A_{n} \stackrel{G}{\rightarrow} A$.

Also let

$$
\hat{L}_{n}(t, x, u)=\int_{z} L_{n}(t, z, x(z), u(z)) d z \text { and } \hat{L}(t, x, u)=\int_{z} L(t, z, x(z), u(z)) d z .
$$

Then from hypothesis $H(L)_{3}$ and theorem 3.1 of Salvadori [34] we have $\hat{L}_{n}(t, \cdot, \cdot) \stackrel{\tau}{\rightarrow} \hat{L}(t, \cdot, \cdot)$ a. e..

Finally set $U_{n}(t)=\left\{u \in L^{2}(Z):\left\|u_{n}\right\|_{L^{2}(Z)} \leqq r_{n}(t)\right\}$ and $U(t)=\left\{u \in L^{2}(Z):\|u\|_{L^{2}(Z)}\right.$ $\leqq r(t)\}$. Since by hypothesis $H(r), r_{n}(t) \rightarrow r(t)$ a. e., it is easy to see that $U_{n}(t)$ $\underset{K}{\rightarrow} U(t)$ a. e.. forms.

Rewrite problems $(* * * *)_{n}^{\prime}$ and $(* * * *)^{\prime}$ in the following equivalent abstract

$$
\left\{\begin{array}{c}
\hat{J}_{n}(x, u)=\int_{0}^{r} \hat{L}_{n}(t, x(t), u(t)) d t \longrightarrow \inf =m_{n} \\
\text { s. t. } \dot{x}(t)+A_{n} x(t)=\int_{0}^{t} \hat{k}(t-s) \hat{f}_{n}(s, x(s)) u(s) d s \text { a. e. } \\
x(0)=x_{0}^{n}(\cdot) \\
u(t) \in U_{n}(t) \text { a. e., } u(\cdot) \text { is measurable }
\end{array}\right\}(* * * *)_{n}^{\prime}
$$

and 


$$
\left\{\begin{array}{l}
\hat{J}(x, u)=\int_{0}^{r} \hat{L}(t, x(t), u(t)) d t \rightarrow \inf =m \\
\text { s. t. } \dot{x}(t)+A x(t)=\int_{0}^{t} \hat{k}(t-s) \hat{f}(s, x(s)) u(s) d s \text { a. e. } \\
x(0)=x_{0}(\cdot) \\
u(t) \in U(t) \text { a. e., } u(\cdot) \text { is measurable }
\end{array}\right\}^{* * * *),}
$$

These have the same forms as problems $(* * *)_{n}^{\prime}$ and $(* * *)^{\prime}$ respectively. So invoking theorem 6.2 , we can have the following result:

THEOREM 7.2. If hypotheses $H(A)_{4}, H(f)_{3}, H(k), H(L)_{3}, H(r)$ hold and $x_{0}^{n}$ $\stackrel{s}{\rightarrow} x_{0}$ in $L^{2}(Z)$, then $m_{n} \rightarrow m$.

AcKNowledgement: The author is deeply indebted to the referee for his many corrections and remarks that improved the content of this paper.

\section{REFERENCES}

[1] T. ANGell, "On the optimal control of systems governed by nonlinear Volterra integral equations" J. Optim. Theory Appl. 19 (1976), pp. 29-45.

[2] R. Ash, "Real Analysis and Probability" Academic Press, New York (1972).

[3] H. Aтtouch, "Variational Convergence for Functions and Operators" Pitman, Boston (1984).

[4] E. BALDER, "Necessary and sufficient conditions for $L^{1}$-strong-weak lower semicontinuity of integral functionals" Nonl. Anal. 11 (1987), pp. 1399-1404.

[5] V. BARBU, "Nonlinear Semigroups and Differential Equations in Banach Spaces" Noordhoff International Publishing, Leyden, The Netherlands (1976).

[6] A. Bressan and G. Colombo, "Extensions and selections of maps with decomposable values" Studia Math XC (1988), pp. 69-86.

[7] F. BROWDER, "Pseudomonotone operators and nonlinear elliptic boundary value problems on unbounded domains" Proc. Nat. Acad. Sci. 74 (1977), pp. 2659-2661.

[8] L. Cesari, "Optimization-Theory and Applications" Springer, New York (1983).

[9] P.V. CHuONG, "Existence of solutions for random multivalued integral equations J. Integral Equations 7 (1984), pp. 143-173.

[10] K. Deimling, "Sample solutions of stochastic ordinary differential equations" Stoch. Anal. Appl. 3 (1985), pp. 15-21.

[11] J. Delahaye and J. Denel, "The continuities of the point to set maps, definitions and equivalences" Math. Programming Study 10 (1979), pp. 8-12.

[12] J. Diestel and J. Uhl, "Vector Measures" Math. Surveys, Vol. 15, A. M.S., Providence, R.I. (1977).

[13] N. Dunford and J. Schwartz, "Linear Operators I" Wiley-Interscience, New York (1958).

[14] G. Edgar, "Measurabi ity in a Banach space II" Indiana Univ. Math. Jour. 28 (1979), pp. $559-579$.

[15] K. Glashoff and J. SPREKels, "The regulation of temperature by thermostats and set valued integral equations" J. Integral Equations 4 (1982), pp. 95-112. 
[16] T. KIFFE, “A Volterra integral equation and multiple valued functions” J. Integral Equations 3 (1981), pp. 93-108.

[17] E. Klein and A. Thompson, “Theory of Correspondences" Wiley, New York (1984).

[18] D. Kravvaritis AND N.S. Papageorgiou, "Multivalued perturbations of subdifferential type evolution equations in Hilbert spaces" J. Diff. Equations 76 (1988), pp. 238-255.

[19] K. Kuratowski, “Topology I” Academic Press, New York (1966).

[20] G. Ladde and V. Lakshmikantham, "Random Differential Inequalities" Academic Press, New York (1980).

[21] V. LEvin, "Borel sections of many valued maps" Siberian Math. Jour. 19 (1978), pp. 434-438.

[22] J.-L. Lions, "Quelques Methodes de Resolution des Problémes aux Limites non Lineaires" Dunod-Gauthier Villars, Paris (1969).

[23] U. Mosco, "On the continuity of the Young-Fenchel transform" J. Math. Anal. Appl. 35 (1971), pp. 518-535.

[24] E. NAGY, "A theorem on compact embedding for functions with values in an infinite dimensional Hilbert space” Ann. Univ. Sci. Budapest 24 (1981), pp. 243245.

[25] A. Nowak, "On generalized random differential equations" Demonstratio Math. 16 (1983), pp. 469-476.

[26] B. Pachpatte, "A note on the Gronwall-Bellman inequality" J. Math. Anal. Appl. 44 (1973), pp. 758-762.

[27] N.S. PApAgeorgiou, “Volterra integral inclusions in Banach spaces" J. Integral Equations and Applications 1 (1988), pp. 65-81.

[28] N.S. PApageorgiou, "Existence and convergence results for integral inclusions in Banach space" J. Integral Equations and Applications 2 (1988), pp. 265-285.

[29] N.S. Papageorgiou, "Random differential inclusions in Banach spaces" J. Differential Equations 65 (1986), pp. 287-303.

[30] N.S. Papageorgiou, "On the theory of Banach space valued multifunctions. Part 1: Integration and conditional expectation" J. Multiv. Analysis 17 (1985), pp. 185-206.

[31] N.S. PAPAgeorgiou, "Convergence theorems for Banach space valued integrable multifunctions" Intern. J. Math. and Math. Sciences 10 (1987), pp. 433-442.

[32] R. Ragimkhanov, "The existence of solutions to an integral equation with multivalued right hand side" Siberian Math. Jour. 17 (1976), pp. 533-536.

[33] R. T. Rockafellar, “Convex Analysis" Princeton University Press, Princeton (1970).

[34] A. Salvadori, "On the M-convergence for integral functionals on $L^{p}(X)$ " Atti Seminario Mat. Fis. Univ. Modena 33 (1984), pp. 137-154.

[35] J. Sokolowski, "Optimal control in coefficients for weak variational problems in Hilbert spaces” Appl. Math. Optim. 7 (1981), pp. 283-293.

[36] H. TANABE, "Equations of Evolution" Pitman, London (1979).

[37] C. Tsokos AND W. PAdgett, "Random Integral Equations with Applications to Life Sciences and Engineering" Academic Press, New York (1974).

[38] D. WAGNER, "Survey of measurable selection theorems" SIAM J. Control and Optim 15 (1977), pp. 859-903.

[39] J. WARGA, “Optimal Control of Differential and Integral Equations" Academic 
Press, New York (1972).

[40] V. Zhikov, S. Kozlov AND O. OleiniK, "G-convergence of parabolic operators" Russian Math. Surveys, Vol. 36 (1981), pp. 9-60.

National Technical University

Department of Mathematics

Athens, 15773, Greece 